\title{
Mathematical Models for Electrostatics of Heterogeneous Media
}

\author{
Toshko Boev \\ Department of Differential Equations, University of Sofia, Sofia, \\ Bulgaria
}

\section{Introduction}

Since most of twenty years intensive investigations, in Physical Chemistry and applied Physics, have been directed to various basic processes for complexly structured material systems, with a strong accent on surface phenomena problems. Such systems, known as heterogeneous, consist typically of different bulk phases, separated by specific interfaces, which can be also realized as containing distinct but near by sub-phases. Additionally, the common line contours, of 2D sub-phases, are treated as materially autonomous (1D) phases as well. One of the main directions in said topics consider surface nucleation phenomena in gas-liquid systems. The interest here has been provoked mainly from the open questions for the mechanism of the surface nucleation - in particular in lipid systems. Said questions essentially concern basic topics of Physical Chemistry, related also to ecological applications. As a second class, note the problems on structure building of semiconductor films of aircrystal media, via an actual technological interest: it primarily concerns the main factors governing the growth and roughness of semiconducting surface films. Another (third) class of related topics is shown by the recent studies on cell biology problems (e.g. [4]). This class includes also mathematical models for detecting of anomalies in the human organic systems - for instance, the blood circulatory system and that of the white liver oxygen transfer.

The electrostatic properties of matter have been taken as the basic framework for investigations of surface phenomena problems. Especially, adequate expressions have been sought for the electric potential - as the key quantity, integrating the basic electrostatic parameters of medium. Our main goal here consists in finding such expressions, primarily concerning the interface potential of complex (3-2-1D) heterogeneous systems. Said aim yields the key question how to construct a proper mathematical model of the matter electrostatics, which introduces a correct problem for the electric potential. Secondly, it is necessary to do the main steps in the mathematical analysis of the relevant problem. Here we propose an answer of the above question, taking into account two required basic steps. The first one consists in introducing the object called heterogeneous media, when in reality we have given a material system of different bulk (3D) phase, for instance - gas and liquid, with a relatively thin transition layer (say emulsion). In our treatment an additional stage of heterogeneity is presumed: the bulk transition layer consists also of two near-by sub-phases (of differing matter). According to the Gibbs idealizing approach ([6]), we have to consider 
said transition layer as a material 2D formation $S$ - the common surface boundary of the two bulk phases. Thus the first stage of introducing heterogeneous media results in the (Gibbs idealized) heterogeneous system $\left\{B^{-} \cup S \cup B^{+}\right\}$, consisting in two bulk phases - $B^{+}$, $B^{-}$, and the $2 \mathrm{D}$ phase $S-$ as an interface. The above mentioned stage (of second order heterogeneity) should be noted as a first point of new elements in our results: we assume the interface in the form $S=\left\{S^{-} \cup l \cup S^{+}\right\}$, with two 2D sub-phases - $S^{+}, S^{-}$(of generally differing 2D materials), and a line material component $l$ - the common boundary of $S^{+}$, $S^{-}$. Component $l$ is assumed homogeneous and introduced by applying again the Gibbs idealizing approach, taken now on the interface. Next, let us comment the second required basic step of modeling. Because the aim is to model electrostatics, we have to deal with the Maxwell electrostatic system, as a constituting-phenomena low, applied however for the totally heterogeneous medium $\left\{B^{-} \cup S^{-} \cup l \cup S^{+} \cup B^{+}\right\}$. Note here the specific detail concerning the charge density $\rho$ (which essentially enters in the Maxwell system): via an electrochemical principle, we should presume $\rho$ depending on the electric potential $u$, i.e. it generally holds $\rho=\rho[u]$. Moreover, function $\rho[u]$ takes the form of the known Boltzmann distribution, for instance in case of electrolytes. Because the Gibbs idealization assumes a step transition across the interface (consequently again such transition, but of lower dimension, is assumed across the phase contour $l$ ), the next appearing problem reads: how to formalize said step transitions, in order to use effectively the Maxwell system. A useful suggestion for the first transition stage (across the interface) can be found in the monograph of D. Bedeaux and J. Vlieger ([2]). According to Bedeaux - Vlieger, we introduce the relevant material characteristics across the interface by a (first level) decomposition scheme of singularities, using Heaviside step functions $\eta^{ \pm}$regarding respectively the bulk phases $B^{ \pm}$, and Dirac delta function $\delta_{s}$, supported on the interface. As a second new point of our modeling, we introduce analogous decomposition scheme on the surface $S$, using in particular delta function supported on the contour $l$. After a technical procedure of solving the Maxwell electrostatic system by singular solutions, it can be established the following final form of our electrostatic model for the class of heterogeneous media $\left\{B^{-} \cup S^{-} \cup l \cup S^{+} \cup B^{+}\right\}$:

$$
\begin{gathered}
\nabla^{2} u=-\varepsilon_{0}^{-1}\left(\varepsilon^{ \pm}\right)^{-1} \rho^{ \pm}[u]\left(\text { in } B^{ \pm}\right) \\
J_{S}[u]+\varepsilon_{s}^{ \pm} \nabla_{S}^{2} u=-\varepsilon_{0}^{-1} \rho_{S}^{ \pm}[u]\left(\text { on } S^{ \pm}\right) \\
J_{l}[u]=-\beta_{l}[u](\text { on } l)
\end{gathered}
$$

Above $u=u(x, y, z)$ is the electric potential, which is sough as bounded continuous function, regular enough in the relevant $3 \mathrm{D}$ and $2 \mathrm{D}$ phases (domains) of the material system; $\nabla^{2}$ is the 3D Laplace operator and $\nabla_{S}^{2}$ is a tangential to surface $S$ Laplace operator; $J_{S}$ is a jump type operator acting on the normal to $S$ derivative of potential $u$, and, by analogy - for operator $J_{l}$, concerning contour $l ; \rho^{ \pm}$and $\rho_{s}^{ \pm}$are the charge density terms, respectively for the bulk $\left(B^{ \pm}\right)$and surface $\left(S^{ \pm}\right)$phases, and $\beta_{l}$ is an analogous quantity, for $1 \mathrm{D}$ phase $l$; $\varepsilon_{0}=8.85 \mathrm{pF} / \mathrm{m}$ is the known absolute dielectric permittivity, $\varepsilon^{ \pm}$and $\varepsilon_{s}^{ \pm}$are the (relative) dielectric permitivities, respectively for the matter of phases $B^{ \pm}$and $S^{ \pm}$, with $\varepsilon_{0}^{-1}=1 / \varepsilon_{0}$, 
$\left(\varepsilon^{ \pm}\right)^{-1}=1 / \varepsilon^{ \pm}$. Mathematically, relations $(0.1)-(0.3)$ present a new type of transmission problem (cf. the Colton - Kress monograph, [3]). In the above generality of formulation, problem (0.1) - (0.3) remains however as open one.

In this chapter we give the main steps of deriving and solve two sub-cases of the general problem (0.1) - (0.3), related to heterogeneous systems with flat interfaces, respectively of semiconductor and organic nature. A straight line contour $l$ enters in both the models as 1D phase of anomalies. Note that the model, with a defect line on the semiconductor interface, is closely related to real experimental data, found by scanning tunneling microscopy. On the other hand, in the case of organic interface (considered in our second model as the known lamina basale), the lamina folio is supposed cleft in two sub-phases by a (straight) line of functionally anomalous intercellular spaces (holes). In both the models bulk charge densities $\rho^{ \pm}[u]$ are replaced with their linear approximations, however charge quantities $\rho_{s}^{ \pm}[u]$ and $\beta_{l}[u]$ enter nonlinearly in the cases, respectively of semiconductor and organic interfaces. By transforming relations (0.2), (0.3), we derive and solve the relevant integral equations for the surface potential $u_{S}$. We obtain also effective formulas for certain approximations of $u_{S}$ (in the case of $S$ - a semiconductor folio), and - for the exact potential (in the case of organic $S$ ). Recall here that the contemporary problems primarily focus in detecting the surface values of the electric potential. As a consequence of finding the surface potential, we express in addition, by the classical Dirichlet problem, the bulk potentials $u^{ \pm}$as well. Thus we get expressions for potential $u$, valid far from the interface, which ensure in particular important diagnostic analyses (made, for instance, by parametric identification inverse problems).

In Sect. 2 we give phenomenology comments and a common derivation of the two considered models. The basic results on the surface potential are presented in Sect. 3. The question for determining of explicit approximations to $u(x, y, z)$ is discussed in Sect. 4, in case of semiconductor interface.

\section{Elements of phenomenology and mathematical modeling}

Let us give firstly some phenomenology comment on said two classes of heterogeneous media. Beginning with the case of organic interface, we should note the following. The interest of tools for biomedical detections of anomalies in the human circulatory system, via the walls-structure of the blood vessels, is directly motivated from the quite specific ruling function of the wall-layers. To recall and clarify the main (simplified) viewpoints here (cf. e.g. [11]), we assume a stretched location of the wall, as the flat surface $(z=0)$ on Fig. 1, below. Said construction is introduced as an admissible version of the real situation: a 3D localization is made to a capillary (practically cylindrical) vessel in the human white liver and the vessel-wall is (functionally) identified with its middle layer (called lamina basale). In reality the wall is a 3D organic threefold layer, deep not less than 120-180 nm and the midmost (just the lamina basale) is of corpulence about $40-60 \mathrm{~nm}$. The upper (external) and lower (internal) layers, built - as a short description - respectively of endothelian and adventitale cells, are neglected. They are considered with a secondary role (compared to lamina basale). As known, acting as a typical bio-membrane of polysaccharide-matter, with 
a fine fibers structure, lamina basale is the main factor for the oxygen transfer to the blood. Via the Gibbs approach (and taking into account the ratio of the wall corpulence to the radius of the capillary vessel, which is $<<1$ ), we consider lamina basale layer as infinitely thin; thus we get an interface film of organic matter in an air-blood (vacuum-blood) heterogeneous 3D media. On the lamina (2D) film it is uniformly distributed a set of points presenting the holes (tunnels, in reality radial to the vessel axis and known as intercellular spaces), which provide the oxygen contact to the blood; they are assumed however of certain functional anomaly, extremely activated on a relatively narrow (cylindrical, in reality) strip, interpreted, following Gibbs, as the middle circumference of the strip, across to the vessel-axis. Thus a specific homogeneous 1D matter phase (of the extreme anomalies) has appeared. Stretching (locally) the curved anomaly-line (and the surrounding cylindrical surface, together), we get the above mentioned (flat-interfaced) construction. Now the organic lamina-film can be presented as the plain $z=0$ (regarding a Cartesian $(x, y, z)$ coordinate system), where said 1D contour, of defective air permeability, has already shaped as a straight line. We shall take this line as the $O y$ - axis. This manner the laminafilm is cleft in two electrostatic equivalent $2 \mathrm{D}$ (sub-) phases by the anomaly contour and we have given a typical case of 3-2-1 D heterogeneous system, schematically shown on Fig. 1, below. The system consists in upper and lower 3D (bulk) phases, respectively of air and liquid, and a complex-structured organic interface (with a special role of a line phase). The bulk phases $B^{+}, B^{-}$fill the subspaces $z>0\left(B^{+}\right), z<0 \quad\left(B^{-}\right)$and their common 2D boundary - the organic interface $S$ - is given (as already noted) by the equation $z=0 ; S$ consists in the two neighbouring surface phases $S^{-}(x<0, z=0), S^{+}(x>0, z=0)$, separated by the anomaly line $l=O y$, as an autonomous phase of $1 \mathrm{D}$ matter. To forecast certain influence of vessel-zones, relatively far from the phase contour $l$, surface phases $S^{-}, S^{+}$are presumed with prescribed asymptotic values $\left(\varphi_{\infty}^{-}, \varphi_{\infty}^{+}\right)$of the electric potential.

It is possible however a sharp variant of anomalies: an air volume can leave involved between the blood and the surface, shaping an internal (lower) air bulk phase. Such presence of two-side bulk air phases is due to the anomalous air transfer: the outgoing stage gets blocked, after a previous air invasion. We would have then a heterogeneous system with upper and lower air (vacuum) bulk phases, a two-phased lamina-interface (as a $2 \mathrm{D}$ film) and a separating the surface phases homogeneous 1D material (straight line) phase.

Another class of heterogeneous media is that including a semiconductor interface. The model under consideration relates to electrostatics for the specific case of air-gas matter, with a semiconductor separating surface (interface), which includes moreover a defect straight line (considered below as the $O y$ - axis, see Fig. 2). In said case of systems the importance of the interface electrostatics is motivated, as already noted, from actual technological questions (e.g. [5], [7]).The structure of such a system can be explained as a space location of given electronic device. For a short description we will take into account the following. By a teen boundary wall of semiconductor-matter it is closed a volume of gas, and the external medium is of air. This boundary, generally curved, will be treated here as flat (observing a small part of it). Thus the system possesses two bulk phases - of internal (gas) and external (air) media, and a flat semiconducting interface, with a fine surface 


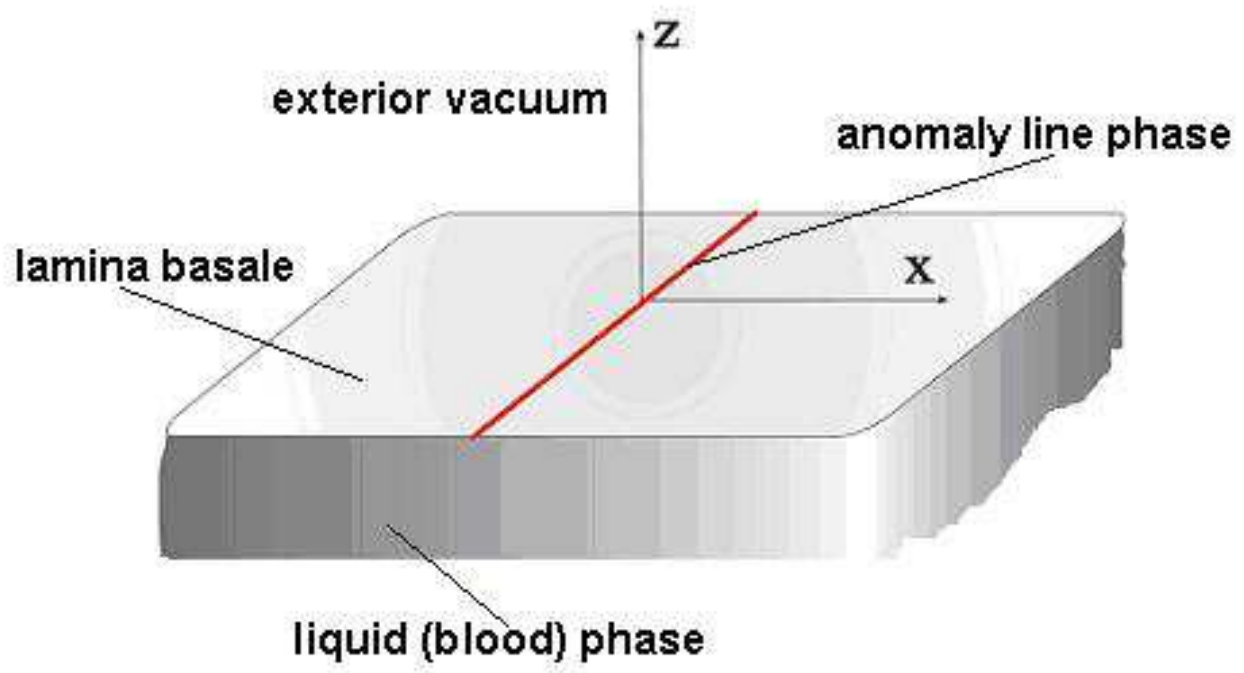

Fig. 1. 
roughness, as a straight line defect. The bulk phases are considered as materially equivalent (3D) sub-domains of vacuum. The separating boundary is of indium-phosphorus, $\operatorname{InP}(110)$, semiconductor: the real corpulence of the $\operatorname{InP}(110)$-wall is neglected and the wall is identified with its external surface film. The defect line, playing the role of a homogeneous detachment, i.e. of an electrostatic autonomous material 1D phase, separates said interface in two surface (2D) phases, denoted as before by $S^{-}(x<0, z=0), S^{+}(x>0, z=0)$. It is posed again a typical case of 3-2-1 D heterogeneity - by a material system, interpreted as vacuum-semiconductor-vacuum (Fig. 2). The $\operatorname{InP}(110)$ surface film is presumed as the plain $z=0$ as well (see Fig. 2) and the $O y$ - axis is oriented on the defect straight line. The vacuum bulk phases fill the upper and lower semi-spaces, $z>0$ and $z<0$, respectively. Each 2D phase (on $z=0$ ) is characterized by an essentially dominating distribution of positively charged phosphorus vacancies, while, as a key anomaly, the line phase $l(l=O y)$ enters in the surface electrostatics symmetrically surrounded by an extremely narrow strip of width $2 d(d>0)$. The whole this band is denuded of phosphorus vacancies ([5], [7]). The above construction is essentially supported by real experimentally found data. A credible visual result of [5] and [7] (see Fig. 2, [7]) has been found by the so-called scanning tunneling microscopy. The picture (Fig. 2, [7]) shows the surface structure, fixed after annealing of $\mathrm{InP}(110)$ samples at temperatures up to $480 \mathrm{~K}$, followed by heat normalizing. The scanned image includes two near by surface domains (let us denote them by $P^{-}, P^{+}$- see the semiplanes $x>d, x<-d$ on Fig. 2, below; clearly $\left.P^{ \pm} \subset S^{ \pm}\right)$. These zones are materially equivalent and separated by a transition strip $T$. Its breadth $(2 d)$ really is less than $10 \mathrm{~nm}$ ([7]). The strip surrounds symmetrically a straight line $l$ (see $O y$, Fig. 2). Each of $P^{-}, P^{+}$is filled by positively charged (+1e) phosphorus vacancies, with about $5.5 \mathrm{~nm}$ ([5]) mean distance between them. Note however that strip $T$ is free of vacancies, but remains generally charged with about $+2 \mathrm{e}$ (per spacing of $0.6 \mathrm{~nm}$, [7]) mean magnitude of charges on the axis of symmetry (the line $l$ ). This way $T$ enters as an electrostatic autonomous surface component. On the other hand the ratio $\left\{\left[\right.\right.$ area] $(T) /$ [area] $\left.\left(P^{-} \cup T \cup P^{+}\right)\right\}$is negligible to consider $T$ as an equipollent (say to $P^{-}, P^{+}$) 2D surface component. Moreover, the two relations - that of $T$-wide $(10 \mathrm{~nm})$ to the above density unit $(5.5 \mathrm{~nm})$, and the other - of possible (averaged) electrostatic impact of $T$ to the influence of its middle axis $l$, allow some identifying of the strip $T$ with the axis $l$; thus $l$ takes the role of an intrinsic 1D phase. Let us note that, via the Gibbs approach, the semi-zones $\left(T^{+}(x>0), T^{-}(x<0)\right)$ of $T$ seem to complicate additionally the surface heterogeneity, imposing - as a (Gibbs) principle - new line phases: the contours $l_{d}^{-}=\{x=-d ; z=0\}$ and $l_{d}^{+}=\{x=d ; z=0\}$. These (new) phases however enter also negligibly in the surface electrostatics: across $l_{d}^{-}, l_{d}^{+}$the surface electric field stays continuous, under equal permitivity-values $\varepsilon_{s}$. So-described picture (of a smooth, flat 2D film) represents a real surface layer with certain nanoscopic roughness, due to step defects. In reality $l$ is actually the edge of a step, deep about $4 \mathrm{~nm}$ ([7], Fig. 1), $P^{-}$ and $P^{+}$are the terraces (lower and upper, say) of the step, and $T$ is a space construction, divided by $l$ in two halves, $T^{-}$(lower) and $T^{+}$(upper), marking off the edge from the relevant terraces.

The next stage of this section is to sketch the basic step of modeling. Via the introduced framework (see Sect. 1) the key tool for description of electrostatic phenomena in complex media relates to the Maxwell system (in case of dielectrics, e.g. [10], [12]): 


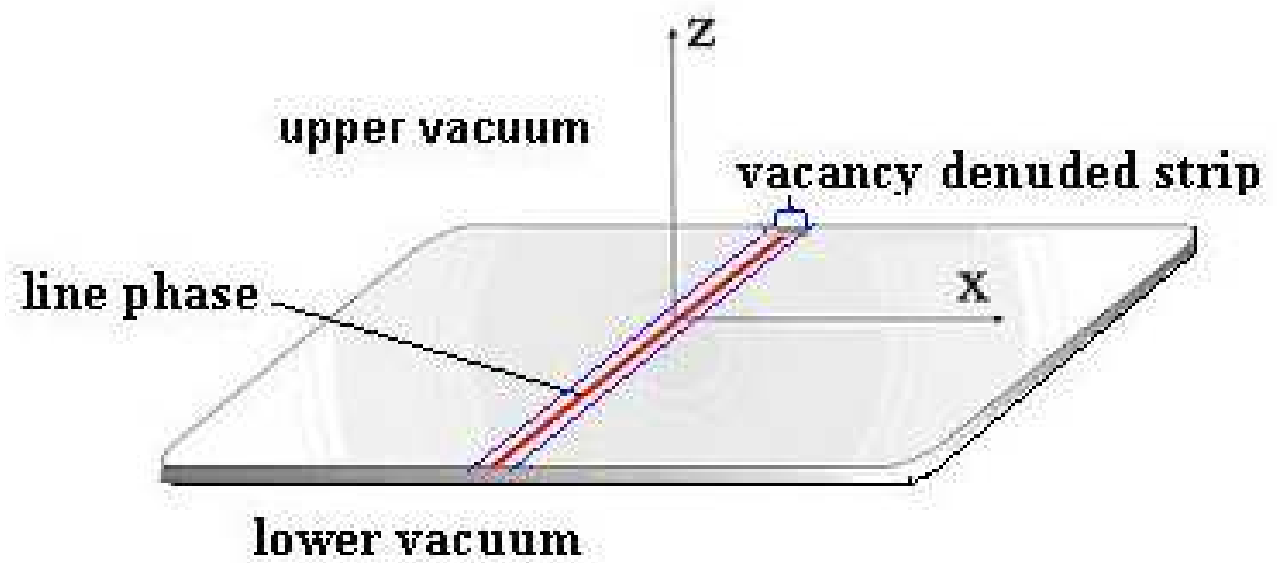

Fig. 2.

a) $\nabla . \mathrm{D}=\rho$; b) $\mathrm{D}=-\varepsilon_{0} \varepsilon \nabla u$.

Here $\nabla$ is the nabla operator, $\mathrm{D}$ is the vector of the electric induction ([12]), called also (in Electrochemistry, e.g. [9]) electric displacement, $\nabla$. D is the formal scalar product of the vectors nabla and $\mathrm{D}$, i.e. $\nabla . \mathrm{D}=\operatorname{div} \mathrm{D} ; \rho$ is the charge density; $\varepsilon$ is the relative dielectric permitivity for the relevant part of the medium (in particular $\varepsilon=\varepsilon_{b}^{-}$, at $z<0, \varepsilon=\varepsilon_{s}$, at $z=0, x \neq 0) ; u$ is the electric potential, $\nabla u=\operatorname{grad}(u)$, where $(-\nabla u)$ represents the electric field, propagated in the whole 3D material system. Equations (2.1) hold for the total (3D) system and, as known, potential $u$ is a continuous function of $(x, y, z)$, in spite of the various material phases; the heterogeneity of the system is indicated however mainly by the 
quantities $\mathrm{D}$ and $\rho$ (note that the permitivity $\varepsilon$ enters in these quantities). Next, from the singular decompositions, mentioned in Sect.1, applied below for quantities D and $\rho$, we get the following problem. Find the (admissibly regular) solutions $(\mathrm{D}, u)$ to $(2.1)$, corresponding to the said singular decompositions.

Both the considered cases of heterogeneous systems are however homogeneous on the $y-$ direction, due to assumed homogeneity of the 1D phase $l$, and the electric potential $u=u(x, y, z)$ will actually depend on $x, z$, i.e. $u=u(x, z)$. Applying systematically a double decomposition scheme in reworking of the Maxwell system (see below), we shall establish the following final formulation to the sought mathematical models:

$$
\begin{gathered}
\nabla^{2} u=\kappa_{b}^{2} u(z \neq 0), x \in R^{1} ; \\
|u| \leq \text { const., }(x, z) \in R^{2} ; \\
u(x,+0)=u(x,-0), x \in R^{1} ; \\
\Delta\left[\varepsilon_{b} u_{z}\right]+\varepsilon_{s} u_{x x}=\varepsilon_{s} k_{s}^{2}\left(u-\varphi_{\infty}\right)+\rho_{s}^{*}[u], x \neq 0 ; \\
u(-\infty, 0)=\varphi_{\infty}^{-}, u(+\infty, 0)=\varphi_{\infty}^{+} ; \\
u(-0,0)=u(+0,0) ; \\
\varepsilon_{s}^{+} u_{x}(+0,0)-\varepsilon_{s}^{-} u_{x}(-0,0)=-\beta_{l}[u] .
\end{gathered}
$$

In (2.2) $\nabla^{2} \equiv \partial_{x}^{2}+\partial_{z}^{2}$ is the Laplace operator; $u_{x}, u_{z}, u_{x x}$ are first or second order derivatives regarding the relevant variable; $u(x,+0), u(x,-0)$ are respectively the limits (supposed finite) $\lim _{z \rightarrow 0} u(x, z) \quad$ (at $z>0$ or $\left.z<0\right)$, and, by analogy - for $u_{z}(x,+0), u_{z}(x,-0)$; $u( \pm 0,0)=\lim _{x \rightarrow 0} u(x, 0)$ and $u_{x}( \pm 0,0)=\lim _{x \rightarrow 0} u_{x}(x, 0)$, respectively at $x>0, x<0$, both - for $u( \pm 0,0)$ and $u_{x}( \pm 0,0) ; u( \pm \infty, 0)=\lim _{x \rightarrow \pm \infty} u(x, 0)$. (Above $R^{m}$ is the real $m$-dimensional Euclidean space, $m=1,2, \ldots$.) As known, parameters $k_{b}, \varepsilon_{b}$ and $k_{s}, \varepsilon_{s}$ are the main factors of the system-electrostatic nature; they are given step constants: $\varepsilon_{b}=\varepsilon_{b}^{+}(z>0), \varepsilon_{b}=\varepsilon_{b}^{-}(z<0)$, $\varepsilon_{b}^{+}, \varepsilon_{b}^{-}$- positive (and generally different); $k_{b}=k_{b}^{+}(z>0), k_{b}=k_{b}^{-}(z<0)$ are nonnegative constants in (2.2); $k_{s}=k_{s}^{+}(x>0), k_{s}=k_{s}^{-}(x<0)$ - in (2.4), with positive $k_{s}^{+}, k_{s}^{-}$; by analogy, $\varepsilon_{s}=\varepsilon_{s}^{+}(x>0), \varepsilon_{s}=\varepsilon_{s}^{-}(x<0)-$ in (2.4), (2.6), with $\varepsilon_{s}^{+}>0, \varepsilon_{s}^{-}>0$ - constants. The material meaning of parameter $k_{s}$ (by analogy from that of $k_{b}$ ) is expressed by the quantity $k_{s}^{-1}=\frac{1}{k_{s}}$, known as the surface Debye length (e.g. [13], or the surface screening length (e.g. [7]). Parameters $\varepsilon_{b}, \varepsilon_{s}$ are respectively the bulk and surface dielectric permitivities, with $\varepsilon_{b}^{+}\left(\varepsilon_{b}^{-}\right), \varepsilon_{s}^{+}\left(\varepsilon_{s}^{-}\right)$- for the relevant bulk and surface phases. The asymptotic values of the 
potential are prescribed by the parameter $\varphi_{\infty}$ (a given quantity): $\varphi_{\infty}=\varphi_{\infty}^{ \pm}($at $x>0, x<0)$, where $\varphi_{\infty}^{+}, \varphi_{\infty}^{-}$are real, generally different constants; the parameter $\beta_{l}=\beta_{l}[u]$ enters as $\beta_{l}=\frac{\rho_{l}}{\varepsilon_{0}}$ by $\rho_{l}=\rho_{l}[u]$ - the electric charge density (supposed depending on potential $u$ ) upon the line phase; $\varepsilon_{0}=8.85 \mathrm{pF} / \mathrm{m}$ is the mentioned absolute dielectric permitivity. In equation (2.4.b) $\Delta\left[\varepsilon_{b} u_{z}\right]=\Delta\left[\varepsilon_{b} u_{z}\right](x, 0) \quad$ is the space-jump operator, $\Delta\left[\varepsilon_{b} u_{z}\right](x, 0) \equiv \varepsilon_{b}^{+} u_{z}(x,+0)-\varepsilon_{b}^{-} u_{z}(x,-0)$ and in the right hand side of (2.4) we have $\rho_{s}[u]=\varepsilon_{0}\left(\varepsilon_{s} k_{s}^{2}\left(u-\varphi_{\infty}\right)+\rho_{s}^{*}[u]\right)$. In the case of organic interface we shall suppose $\rho_{s}^{*}[u]=0$, while for semiconductors we shall use $\rho_{s}^{*}[u] \equiv \omega_{d}^{0}\left(\varepsilon_{s} k_{s}^{2} \frac{\Delta \varphi_{\infty}}{2} s g-q_{s}[u]\right)$. Here $\omega_{d}^{0}=\omega_{d}^{0}(x) \equiv \frac{1}{2 d} \omega^{0}\left(\frac{x}{d}\right)$ is the rescaled characteristic function of the unit interval, i.e. $\omega^{0}(x) \equiv 1,|x|<1 ; \omega^{0}(x) \equiv 0,|x|>1 ; \quad \Delta \varphi_{\infty} \equiv \varphi_{\infty}^{+}-\varphi_{\infty}^{-} \quad$ is the asymptotic surface power; $s g=s g(x)=\frac{x}{|x|}$ is the sign function; $q_{s}[u] \equiv \frac{1}{2 !}\left[\varepsilon_{s} k_{s}^{2}\left(u-\varphi_{\infty}^{*}\right)\right]^{2}-\frac{1}{3 !}\left[\varepsilon_{s} k_{s}^{2}\left(u-\varphi_{\infty}^{*}\right)\right]^{3}$, where $\varphi_{\infty}^{*}=\frac{\varphi_{\infty}^{+}+\varphi_{\infty}^{-}}{2}$. The cubic nonlinear charge density (of the surface $z=0$ ) is preferred just on the strip $|x|<d$ - by the rest term $\omega_{d}^{0} q_{s}[u]$, while a linear approximation (regarding potential $u$ ) is assumed adequate to reality out of the strip.

Via the phenomenology-essence potential $u$ will be searched for a bounded function (condition (2.3)), continuous in $R^{3}$, classically regular in the sets $z \neq 0$ and $x \neq 0(z=0)$, with continuous gradients $u_{z}, u_{x}$, respectively at $z \geq 0, z \leq 0$ (for $u_{z}$ ), and $x \leq 0, x \geq 0(z=0)-$ for $u_{x}$. Now we can define the needed space of regular solutions to (2.2) - (2.6).

Definition. A function $u(x, z)$, with the above noted regularity, shall be called classical solution to problem (2.2) - (2.6) if satisfies the additional property $u(x, 0)-\varphi_{\infty} \in L_{2}$ and relations (2.2) - (2.6). ( $L_{2}$ is the well known space of the squared-integrable functions.)

From the vacuum assumption for the upper (external) air phase $(z>0)$ we suppose from now on:

$$
\varepsilon_{b}^{+}=1, k_{b}^{+}=0 .
$$

The central results below relate to determination of the surface potential (possibly by an explicit approximation) - as the key first step in solving the full (2.2) - (2.6) - problem.

Let us now sketch the main steps for derivation of the final mathematical models, starting from the Maxwell system (2.1). Via the presumed complex heterogeneity, we shall seek solutions $(\mathrm{D}, u)$ of system (2.1) by decompositions in two levels (bulk and surface), of the following type:

a) $\rho=\rho_{b}^{-} \eta^{-}+\rho_{b}^{+} \eta^{+}+\rho_{s} \delta(z) ;$ b) $\rho_{s}=\rho_{s}^{-} \eta_{s}^{-}+\rho_{s}^{+} \eta_{s}^{+}+\rho_{l} \delta_{l}$; 


$$
\begin{gathered}
\mathrm{D}=\left(\mathbf{D}_{b}\right)^{-} \eta^{-}(z)+\left(\mathbf{D}_{b}\right)^{+} \eta^{+}(z)+\mathrm{D}_{s} \delta(z), \\
\mathrm{D}_{s}=\left(\mathbf{D}_{s}\right)^{-} \eta_{s}^{-}+\left(\mathbf{D}_{s}\right)^{+} \eta_{s}^{+}+\mathrm{D}_{l} \delta_{l} .
\end{gathered}
$$

In the above relations $\eta^{+}(z) / \eta^{-}(z)$ are respectively the Heaviside forward/backward functions (i.e. $\eta^{+}(z)=1$, at $z>0, \eta^{+}(z)=0$, at $\left.z<0, \eta^{-}(z) \equiv \eta^{+}(-z)\right)$ and $\delta(z)$ is the Dirac delta-function, supported at $z=0 ; \eta_{s}^{-}=1$, at $z=0, x<0$ and $\eta_{s}^{-}=0$, at $z=0, x>0$, by analogy: $\eta_{s}^{+}=1$, at $z=0, x>0$ and $\eta_{s}^{+}=0$, at $z=0, x<0$; next, $\delta_{l}$ is delta-function, supported on the line $l: x=0(z=0)$, and we shall also use the notation $\delta(x)$, for $\delta_{l}$. Relations (2.8.a), (2.8.b) and (2.9), (2.10) just illustrate, respectively for the charge density and the electric induction, the essential generalization, in two levels (see [13]), of the BedeouxVlieger ([2]) step formalism to the bulk-surface-bulk transitions. Remark: terms like $\rho_{b}^{ \pm} \eta^{ \pm}(z)$ do not enter in the right hand side of (2.8.a) in the case of semiconductor interface because of the vacuum hypothesis $\left(\rho_{b}^{-}=\rho_{b}^{+}=0\right)$. In (2.9) $\left(\mathbf{D}_{b}\right)^{-}$and $\left(\mathbf{D}_{b}\right)^{+}$are at least smooth (vector) functions of $(x, z)$, respectively at $z<0$ and $z>0$, with finite but generally different limit values at $z \rightarrow 0, \forall$ fixed $x$ and $\mathrm{D}_{s}$ is a vector function of $x$, assumed in the form of (2.10). Analogous presumptions hold to $\left(\mathbf{D}_{s}\right)^{-},\left(\mathbf{D}_{s}\right)^{+}-$in (2.10), as functions actually of $x$ (with finite and different limit values at $x \rightarrow 0$ ), and to (scalar) functions $\rho_{s}^{ \pm}=\rho_{s}^{ \pm}(x)$ (considered as at least continuous respectively at $x \leq 0$ and $x \geq 0) ; \rho_{l}$ and $\mathrm{D}_{l}$ enter in (2.8.b) and (2.10) respectively as constant scalar and vector. Substituting from (2.8) - (2.10) into electrostatic equations (2.1), we get (with $\varepsilon_{b}^{+}=1$ ):

$$
\begin{gathered}
\nabla .\left(\mathbf{D}_{b}\right)^{+}=0(z>0), \nabla \cdot\left(\mathbf{D}_{b}\right)^{-}=\rho_{b}^{-}[u](z<0) ; \\
\left(\mathbf{D}_{b}\right)^{+}=-\varepsilon_{0} \varepsilon_{b}^{+} \cdot \nabla u(z>0),\left(\mathbf{D}_{b}\right)^{-}=-\varepsilon_{0} \varepsilon_{b}^{-} \cdot \nabla u(z<0) ; \\
D_{+}^{z}(x, 0)-D_{-}^{z}(x, 0)+\nabla_{s} . \mathrm{D}_{s}=\rho_{s}[u](z=0, x \neq 0) ; \\
\mathrm{D}_{s}=-\varepsilon_{0} \varepsilon_{s} \cdot \nabla_{s} u(z=0, x \neq 0) ; \\
\left(\mathbf{D}_{s}\right)^{x,+}-\left(\mathbf{D}_{s}\right)^{x,-}=\rho_{l} .
\end{gathered}
$$

Here we have denoted by $\nabla_{s}$ the tangential (to $z=0$ ) component of the nabla operator $\nabla$; $D_{+}^{z}(x, 0)=\lim _{z} D^{z}(x, z)$, and, by analogy - for $D_{-}^{z}(x, 0)$, where $D^{z}$ is the normal to $z=0$ component of vector $\mathrm{D}$, and the limits are supposed finite, $\forall x ;\left(\mathbf{D}_{s}\right)^{x,-},\left(\mathbf{D}_{s}\right)^{x,+}$ are the relevant limits (also assumed finite), at $x \neq 0$, for the normal to $l$ component $\left(\mathbf{D}_{s}\right)^{l}$ of $\mathbf{D}_{s}$. Let us note (calculating the results of said substituting) that the normal to $z=0$ component $D_{s}^{z}$ of vector $D_{s}$ is found to vanish $\left(D_{s}^{z}=0\right)$, i.e. $D_{s}$ presents a flat (planar) vector field (see e.g. [13] for details). Remark: the used derivations of the Heaviside and Dirac delta-functions, necessary to get system (2.11) - (2.13), are taken in the Schwartz distributions meaning (e.g. [8]).

Now we will discuss the charge density terms $\rho_{b}^{-}[u], \rho_{s}[u]$, respectively in (2.11.a), (2.12.a), especially that of $\rho_{s}$. For the vacuum-semiconductor-vacuum systems we have to take $\rho_{b}^{-}[u]=0$ (from the vacuum hypothesis). For vacuum-lamina-liquid systems it is 
established certain relation $\rho=\rho[u]$. Such type of dependence is well known for electrolytes by the Gouy-Chapmann theory, where $\rho[u]$ is expressed by the so-called Boltzmann distribution (see e.g. [9]) for the bulk phases, and in the case of organic interface we can replace in (2.11.a) $\rho[u]$ with its linear approximation $-\varepsilon_{0} \varepsilon_{b} k_{b}^{2} u$. Via the real phenomena, the surface charges should also depend on the space variables by potential $u$, i.e. $\rho_{s}=\rho_{s}[u]$. A preliminary motivation to do that follows from the argument that the polysaharide matter of the interface admits to consider it as a lipid medium, where the potential-magnitude can be assumed relatively smaller than the basic ratio $\left(R T_{0}\right) / F$, which yields that linear approximations become acceptable $\left(F, R, T_{0}\right.$ are - as follows - the so-called Faraday and gas constants, and the absolute temperature). The Boltzmann principle, applied for surfaces, suggests dependence $\rho_{s}=\rho_{s}\left[u-\varphi_{\infty}\right]$ to the surface phases; i.e. we can take again the relevant linear approximation $-\varepsilon_{0} \varepsilon_{s} k_{s}^{2}\left(u-\varphi_{\infty}\right)$ instead of $\rho_{s}\left[u-\varphi_{\infty}\right]$. In the case of semiconductors however a nonlinear dependence $u \rightarrow \rho_{s}[u]$ could be derived from a parametric expression, known as Fermi-Dirac integral (e.g. [1]). Said dependence is of exponential type regarding the potential and relates well enough to the simpler one, $\rho=\varepsilon_{0} \exp \left(-\varepsilon k^{2} u\right)$, used for the so-called screened Coulomb potential in the bulk phases (see [5], [7] and the literature therein). It is important that the same expression has been experimentally examined in [5], [7] (with $\varepsilon_{s}, k_{s}$ instead respectively of $\varepsilon, k$ ), to the analysis for the surface density of phosphorus vacancies. From the above-noted viewpoint we shall chose a truncation of exponential dependence for the surface phases in the following form (taking into account the total electro-neutrality of the considered material system and the specific inclusion of component $T$ ):

$$
\rho_{s}=\varepsilon_{0}\left(-\varepsilon_{s} k_{s}^{2}\left(u-\varphi_{\infty}\right)-\omega_{d}^{0} \varepsilon_{s} k_{s}^{2} \frac{\Delta \varphi_{\infty}}{2} s g+\omega_{d}^{0} q_{s}[u]\right) .
$$

To get the above expression for $\rho_{s}$ (in said framework) we start from the following type of exponential dependence for the surface phases: $\rho_{s}=\varepsilon_{0}\left(\exp \left[-\varepsilon_{s} k_{s}^{2}\left(u-\varphi_{\infty}\right)\right]-1\right)$. Under the behaviour $\rho_{s}[t] \sim t$, at $t \approx 0$, such an expression takes into account the total electroneutrality of the considered material system, via the upper and lower vacuum phases (by analogy to the case of gas-lamina-liquid media). The difference $u-\varphi_{\infty}$ is present in the exponential term from the assumption to have given asymptotic values $\varphi_{\infty}$ of the surface potential, different from zero (far from the specific edge $l$ ). To forecast the more complicated impact of the vacancy-denuded zone $T^{-} \cup T^{+}$, with $T^{-}=\{-d<x<0 ; z=0\}$ and $T^{+}=\{0<x<d ; z=0\}$, we introduce the modified dependence:

$$
\rho_{s}=\varepsilon_{0}\left\{\left(1-\omega_{d}^{0}\right)\left(\exp \left[-\varepsilon_{s} k_{s}^{2}\left(u-\varphi_{\infty}\right)\right]-1\right)+\omega_{d}^{0}\left(\exp \left[-\varepsilon_{s} k_{s}^{2}\left(u-\varphi_{\infty}^{*}\right)\right]-1\right)\right\} .
$$

When rewrite the above as $\rho_{s}=\left(1-\omega_{d}^{0}\right) \rho_{s}^{0}+\omega_{d}^{0} \rho_{s}^{1}$ (for the sake of shortness), we shall deal with the linear approximation of density $\rho_{s}^{0}$, instead. For undertaking that, the basic motivation issues from the observation on the interaction energy between phosphorus vacancies on the surface (see [7]) - this energy seems to be relatively small. (It has been estimated in [7] with a maximal value of $65 \pm 15 \mathrm{meV}$ at a vacancy separation of $1.2 \mathrm{~nm}$.) On the other hand we cut off the infinite exponential sum for $\rho_{s}^{1}$ up to the cubic term, 
assuming secondary the impact of the higher powers. Thus we shall presume in relation (2.12.a) the given one in (2.14), for the surface charge density of semiconductors. On the linear (1D) phase, the contour $l=O y$, we assume $\rho_{l}=\varepsilon_{0} \beta_{l}$, with $\beta_{l}$ - given constant, for the semiconductor case, while in the organic case we prefer a nonlinear Boltzmann type model $\rho_{l}=\rho_{l}[u]$, forecasting possible unknown complications, close to the line contour.

The next main step of modeling consists in some reworking to system (2.11) - (2.13). By the right hand sides from (2.11.b) we firstly express $\left(\mathbf{D}_{b}\right)^{+},\left(\mathbf{D}_{b}\right)^{-}$in (2.11.a) and come to the Helmholtz-Laplace equations from (2.2). As noted, condition (2.3) corresponds to the physical nature of the potential (to be a space-bounded and continuous quantity). Going to the next relations, (2.4.a), (2.6.a), they show that potential stays continuous across the transition surfaces and lines. On the other hand condition (2.5) introduces the asymptotic value of the surface potential $u(x, 0)$ - they are considered as experimentally known (gauged) data. Afterwards we replace $\mathrm{D}_{s}$ in (2.12.a) by the right hand side of (2.12.b) and use that $D_{+}^{z}(x, 0)=-\varepsilon_{0} \varepsilon_{b}^{+} u_{z}(x,+0), D_{-}^{z}(x, 0)=-\varepsilon_{0} \varepsilon_{b}^{-} u_{z}(x,-0)$ (with $\varepsilon_{b}^{+}=1$, see (2.7)). In addition we rearrange the right hand side of (2.12.a) respectively by the nonlinear density (2.8) or the linear expression $-\varepsilon_{0} \varepsilon_{s} k_{s}^{2}\left(u-\varphi_{\infty}\right)$. This way we get, from (2.12), the complicated jump condition (2.4.b). For the second jump-condition on the electric field (see (2.6.b)), it is enough to recall that $\rho_{l}=\varepsilon_{0} \beta_{l}$ and $\left(\mathbf{D}_{s}\right)^{x, \pm}=-\varepsilon_{0} \varepsilon_{s} u_{x}( \pm 0,0)$.

This completes the sketch of derivation to final form (2.2) - (2.6) of our mathematical models.

\section{The basic integral equation and finding of surface potentials}

We shall reduce in this section problem (2.2) - (2.6) to a corresponding (nonlinear) integral equation - as a background for finding of explicit type presentations to the surface electric potential. Recall firstly the supposed electrostatic equivalence of the surface phases, which yields that $\varepsilon_{s}^{-}=\varepsilon_{s}^{+}=\varepsilon_{s}, k_{s}^{-}=k_{s}^{+}=k_{s}$.

For the needed technical reworks the $x$ - Fourier transformation is systematically taken into account below - by well known conventional expressions (e.g. as in [8]). By the $x$-Fourier transformation to the relations in (2.2) we find ordinary differential equations (regarding $z$ ), which yield the following presentation (for a classical solution $u=u(x, z)$ to problem (2.2) (2.6)):

$$
\hat{u}(\xi, z)=\hat{\varphi}(\xi) \exp (-z|\xi|), z>0 ; \hat{u}(\xi, z)=\hat{\varphi}(\xi) \exp \left(z \sqrt{\xi^{2}+\kappa_{b}^{2}}\right), z<0
$$

It is denoted here by $\hat{u}(\xi, z)$ the (partial) Fourier transformation of $u(x, z)$ - with respect to $x$.

In (3.1) $\varphi(x)=u(x, 0)$ and $\hat{\varphi}$ is the Fourier image of $\varphi$. The jump term in (2.4.b) can be then expressed in the next form:

$$
u_{z}(x,+0)-u_{z}(x,-0)=L[\varphi] ; \hat{L}[\varphi](\xi)=-\lambda(\xi) \hat{\varphi}(\xi), \lambda(\xi)=|\xi|+\varepsilon_{b} \sqrt{\xi^{2}+k_{b}^{2}}
$$


(Above $\hat{L}[\varphi]$ is the Fourier image of $L[\varphi]$ and $\varepsilon_{b}=\varepsilon_{b}^{-}, k_{b}=k_{b}^{-}$.) Thus said jump term is presented as a linear operator $L: \varphi \rightarrow L[\varphi]$, acting from $L_{2}\left(R^{1}\right)$ into the Sobolev space $H^{-1}\left(R^{1}\right)$ (we refer e.g. to [8], for the $H^{k}$-spaces of Sobolev). It admits to separate the key part (2.4) - (2.6) from the full system (2.2) - (2.6) in an autonomous boundary transmission problem:

$$
\begin{gathered}
L[\varphi]+\varepsilon_{s} \varphi^{\prime \prime}=\varepsilon_{s} k_{s}^{2}\left(\varphi-\varphi_{\infty}\right)+\rho_{s}^{*}[\varphi], x \neq 0 ; \\
\varphi( \pm \infty)=\varphi_{\infty}^{ \pm}, \varphi(+0)=\varphi(-0) ; \\
\varepsilon_{s}\left[\varphi^{\prime}(+0)-\varphi^{\prime}(-0)\right]=-\beta_{l} .
\end{gathered}
$$

We have denoted by $\varphi^{\prime}$ and $\varphi^{\prime \prime}$ respectively the first and second derivative of $\varphi(x)$, and by $\varphi( \pm \infty), \varphi(+0), \varphi(-0), \varphi^{\prime}(+0), \varphi^{\prime}(-0)$ - the relevant limits. Taking the substitution $\psi(x) \equiv \varphi(x)-\varphi_{\infty}$, the problem (3.3) reduces into a simpler one for $\psi$. Let us express firstly the quantity $L[\varphi]$ by $L[\psi]$; using notations $\varphi_{\infty}^{*}=\frac{\varphi_{\infty}^{+}+\varphi_{\infty}^{-}}{2}$ and $\Delta \varphi_{\infty}=\varphi_{\infty}^{+}-\varphi_{\infty}^{-}$, we get $L[\varphi]=L[\psi]+\varphi_{\infty}^{*} \cdot L[1]+\frac{\Delta \varphi_{\infty}}{2} \cdot L[s g]$. It is directly seen that $L[s g](x)=2 \sigma_{0}(x)$, where $\sigma_{0}(x)$ : $\hat{\sigma}_{0}(\xi)=i . \operatorname{sg}(\xi) \quad(i=\sqrt{-1})$, and $L[1]=0$. Now, from (3.3), we get the next reduced problem for auxiliary function $\psi$ :

$$
\begin{gathered}
\psi^{\prime \prime}-k_{s}^{2} \psi=-\frac{1}{\varepsilon_{s}}\left(L[\psi]+\Delta \varphi_{\infty} . \sigma_{0}-\rho_{s}^{*}\right), x \neq 0\left(\rho_{s}^{*}=\rho_{s}^{*}\left[\psi+\varphi_{\infty}\right]\right) ; \\
\varepsilon_{s}\left[\psi^{\prime}(+0)-\psi^{\prime}(-0)\right]=-\beta_{l} .
\end{gathered}
$$

The posed $\psi$-problem ((3.4)-(3.5)) is considered on the space of the real functions $\psi$, which are continuous in $(-\infty,-0],[+0,+\infty)$, tend to zero, at $|x| \rightarrow \infty$ and belong to $L_{2}\left(R^{1}\right)$; in addition they are assumed to have the classical regularity at $x \neq 0$, with finite values of the limits $\psi^{\prime}( \pm 0)$. To our next step, observe before that, given a solution $\psi$ of $(3.4)$ - from the said class, we actually have a suitably regular and bounded solution to the equation:

$$
w^{\prime \prime}-k_{s}^{2} w=-\frac{1}{\varepsilon_{s}} F_{s}[\psi], x \neq 0 \quad\left(F_{s}[\psi] \equiv L[\psi]+\Delta \varphi_{\infty} \cdot \sigma_{0}-\rho_{s}^{*}\right) .
$$

Multiplying the Fourier image of $F_{s}[\psi]$ by factor $\left(\xi^{2}+k_{s}^{2}\right)^{-1}$ we find a single bounded solution of (3.6) (see below for some details). Denote this solution by $\frac{1}{\varepsilon_{s}} U_{s}[\psi]$. Then from the general formula for the (bounded) solutions of (3.6), we can directly get a presentation in the form: $\psi(x)=c \cdot \exp \left(-k_{s}|x|\right)+\frac{1}{\varepsilon_{s}} U_{s}[\psi]$ (at $x \neq 0$ ), with a constant $c$. To clarify the structure of $U_{s}[\psi]$, let us introduce the following auxiliary functions, related to the relevant components of operator $F_{s}[\psi]$ : 


$$
\begin{gathered}
g(x)=-\frac{1}{\pi} \int_{0}^{\infty} \frac{\sin (x \xi)}{k_{s}^{2}+\xi^{2}} d \xi ; \\
W_{L}[\psi](x)=\frac{1}{2 k_{s}} L[\psi]^{*} \exp \left(-k_{s}|\cdot|\right)(x) ; \\
R_{s}^{\infty}[\psi](x)=\frac{1}{2 k_{s}} \rho_{s}^{*}\left[\psi+\varphi_{\infty}\right]^{*} \exp \left(-k_{s}|\cdot|\right)(x) .
\end{gathered}
$$

Above $F^{*} \Phi$ is the convolution of two (Schwartz) distributions, $F$ and $\Phi$ (see e.g. [8]), and $W_{L}[\psi](x)$ is a bounded function. In addition $R_{s}^{\infty}[\psi](x)$ is also a bounded function (because $\left(\omega_{d}^{0} s g\right)(x)$ and $\left(\omega_{d}^{0} q_{s}\left[\psi+\varphi_{\infty}\right]\right)(x)$ are compactly supported, while $g(x)$ is evidently bounded. Now, for $U_{s}[\psi]$, it can be found: $U_{s}=W_{L}[\psi]+\Delta \varphi_{\infty} \cdot g-R_{s}^{\infty}[\psi]$ and therefore function $\psi$ (the solution of (3.4)) satisfies the equation:

$$
\psi=c \cdot \exp \left(-k_{s}|\cdot|\right)+\frac{1}{\varepsilon_{s}}\left(W_{L}[\psi]+\Delta \varphi_{\infty} \cdot g-R_{s}^{\infty}[\psi]\right) .
$$

It can be easily seen that the Schwartz derivative of $U_{s}[\psi]$ is in $L_{2}\left(R^{1}\right)$ and that of $c \exp \left(-k_{s}|x|\right)$ is in $H^{-1}\left(R^{1}\right)$, belonging in addition to $L_{2}(x<0), L_{2}(x>0)$. Consequently, differentiating in (3.10), we conclude that $\psi^{\prime} \in H^{-1}\left(R^{1}\right)$ and $\psi^{\prime} \in L_{2}(x \neq 0)$. This yields the next distribution-relation: $\psi^{\prime}(x)=\psi_{1}(x)-\Delta \varphi_{\infty} \delta(x) \quad$ (where $\psi_{1} \in L_{2}\left(R^{1}\right)$ and $\delta(x)$ is the Dirac-function). Then $\left(W_{L}[\psi]\right)^{\prime}(x)=W_{L}\left[\psi^{\prime}\right](x)=W_{L}\left[\psi_{1}\right](x)-\Delta \varphi_{\infty} W_{L}[\delta](x)$. On the other hand - for the rest components of $U_{s}[\psi]$ - it is not difficult to get as follows: $g^{\prime}(x)=W_{L}[\delta](x) ;\left(R_{s}^{\infty}[\psi]\right)^{\prime}(+0)=\left(R_{s}^{\infty}[\psi]\right)^{\prime}(-0)$. Thus we find, for derivative $\psi^{\prime}($ at $x \neq 0)$ :

$$
\psi^{\prime}(x)=-c k_{s} s g(x) \exp \left(-k_{s}|x|\right)+\frac{1}{\varepsilon_{s}}\left(W_{L}\left[\psi_{1}\right](x)+\left(R_{s}^{\infty}[\psi]\right)^{\prime}(x)\right)
$$

However it also holds $W_{L}\left[\psi_{1}\right](+0)=W_{L}\left[\psi_{1}\right](-0)$ and, substituting from (3.11) in the jump relation (3.5), we determine the free constant $c$, as $c=c_{s}$, with

$$
c_{s}=\frac{1}{2}\left(\frac{\beta_{l}}{\varepsilon_{s} k_{s}}-\Delta \varphi_{\infty} \cdot s g(x)\right) .
$$

In order to modify (3.10) into an integral equation regarding the surface potential $\varphi$, we introduce also the next two functions:

$$
\psi_{s}^{0}(x)=\frac{1}{\pi} \int_{0}^{\infty} \frac{\cos (x \xi) d \xi}{\lambda(\xi)+\varepsilon_{s}\left(k_{s}^{2}+\xi^{2}\right)} ; \psi_{s}^{*}(x)=-\frac{1}{\pi} \int_{0}^{\infty} \frac{\sin (x \xi) d \xi}{\lambda(\xi)+\varepsilon_{s}\left(k_{s}^{2}+\xi^{2}\right)} .
$$

Now, going back to (3.10), with $c=c_{s}$ (see (3.12)), via formulas (3.7) - (3.9) and relation $\varphi=\psi+\varphi_{\infty}$, we shall obtain the basic integral equation for surface potential $\varphi$. As a preliminary step we apply the inverse operator of $I-\frac{1}{\varepsilon_{s}} W_{L}$ ( $I$ - the identity) to equation 
(3.10), using that $\varepsilon_{s}\left(k_{s}^{2}+\xi^{2}\right)\left[\lambda(\xi)+\varepsilon_{s}\left(k_{s}^{2}+\xi^{2}\right)\right]^{-1}$ is the Fourier transform of the inverse operator. Reworking this way (3.10) we get the following expression, via the functions from (3.13):

$$
\varphi-\varphi_{\infty}+\rho_{s}^{*}[\varphi]^{*} \psi_{s}^{0}=\beta_{l}[\varphi] \psi_{s}^{0}+\varepsilon_{s} \Delta \varphi_{\infty} \cdot \psi_{s}^{0,1}+\Delta \varphi_{\infty} \cdot \psi_{s}^{*} .
$$

Above $\psi_{s}^{0,1}=\psi_{s}^{0,1}(x)$ is the (Schwartz) first order derivative of function $\psi_{s}^{0}$ (playing a key role, together with $\psi_{s}^{*}$, for the behaviour of potential $\varphi$ ). Note that $\psi_{s}^{0}$ is the (unique) solution of the linear canonical version of problem (3.4), (3.5) (with $\Delta \varphi_{\infty}=0, \beta_{l}=1$ ); this admits, differentiating as before (3.10) (at $\Delta \varphi_{\infty}=0$, with $\psi_{s}^{0}$ instead of $\psi$ ) to use - for the analysis of $\psi_{s}^{0,1}$ - the integral relation:

$$
\psi_{s}^{0,1}(x)=\frac{1}{\varepsilon_{s}}\left(W_{L}\left[\psi_{s}^{0,1}\right](x)-\frac{s g(x)}{2} \exp \left(-k_{s}|x|\right)\right) .
$$

(In particular (3.15) yields the finite limits $\varepsilon_{s} \psi_{s}^{0,1}(+0)=-\frac{1}{2}$ and $\varepsilon_{s} \psi_{s}^{0,1}(-0)=\frac{1}{2}$.)

Equation (3.14) is the sought basic integral equation related to problem (2.2) - (2.6). In the case of vacuum - liquid heterogeneous system, with organic interface, (3.14) takes the specialized form (with $\left.\rho_{s}^{*}[\varphi]=0\right)$ :

$$
\varphi-\varphi_{\infty}=\beta_{l}[\varphi] \psi_{s}^{0}+\varepsilon_{s} \Delta \varphi_{\infty} \cdot \psi_{s}^{0,1}+\Delta \varphi_{\infty} \cdot \psi_{s}^{*} .
$$

We shall study the above equation for nonlinear functions $\beta_{l}[t]=\alpha_{l} t^{3}$, with coefficient $\alpha_{l}>0$. The main results for said class of charge densities are summarized in the following assertion. Below we shall use the quantity $p_{s, 0}=\psi_{s}^{0}(0)$, i.e. $p_{s, 0}=\frac{1}{\pi} \int_{0}^{\infty} \frac{d \xi}{\lambda(\xi)+\varepsilon_{s}\left(k_{s}^{2}+\xi^{2}\right)}$.

\subsection{Proposition}

For arbitrary non zero asymptotic mean value $\varphi_{\infty}^{*}$ of the surface potential, arbitrary parameters $\varepsilon_{b}^{-}>0, k_{b}^{-} \geq 0, \varepsilon_{s}>0, k_{s}>0$, and coefficient $\alpha_{l}: \alpha_{l}>\frac{4}{27} p_{s, 0}^{-1}\left|\varphi_{\infty}^{*}\right|^{-2}$, there exists a unique continuous bounded potential $\varphi(x)$, satisfying (3.16), such that $\varphi_{\infty}^{*} \varphi(0)<0$, determined by the formula

$$
\varphi(x)=\varphi_{\infty}+\beta_{l}\left[t_{0}\right] \psi_{s}^{0}(x)+\varepsilon_{s} \Delta \varphi_{\infty} \cdot \psi_{s}^{0,1}(x)+\Delta \varphi_{\infty} \cdot \psi_{s}^{*}(x), x \neq 0 ;
$$

$t_{0}$ is either the positive (at $\left.\varphi_{\infty}^{*}<0\right)$ or negative (at $\varphi_{\infty}^{*}>0$ ) root of the equation $p_{s, 0} \alpha_{l} t^{3}-t+\varphi_{\infty}^{*}=0$. In addition, the relevant space potential $u(x, z)$ (with $\left.u(x, 0)=\varphi(x)\right)$ is determined as the unique (classical) solution of problem (2.2) - (2.6), by the next formulas:

$$
u(x, z)=\frac{1}{\pi} \int_{-\infty}^{+\infty} \varphi(x-t) \frac{z}{z^{2}+t^{2}} d t, z>0, x \in R^{1}
$$




$$
u(x, z)=\frac{1}{\pi} \int_{-\infty}^{+\infty} \varphi(x-t) \frac{(-1) z}{z^{2}+t^{2}} K_{1}^{0}\left(\kappa_{b} \sqrt{z^{2}+t^{2}}\right) d t, z<0, x \in R^{1} .
$$

(Above $K_{1}^{0}(x) \equiv \pi x K_{1}(x)$, where $K_{1}(x)$ is the McDonald function.)

Proof. Suppose $\varphi(x)$ is a real, continuous solution of (3.16) and let for instance $x \rightarrow+0$ (in (3.16)), using that $\varepsilon_{s} \psi_{s}^{0,1}(+0)=-\frac{1}{2}\left(\right.$ see (3.15)). Because $\beta_{l}[\varphi]=\beta_{l}[\varphi(0)]$, we get then relation $\psi_{s}^{0}(0) \alpha_{l} \varphi^{3}(0)-\varphi(0)+\varphi_{\infty}^{*}=0$, i.e. $t=\varphi(0)$ is (by necessity) a real solution of the algebraic equation $p_{s, 0} \alpha_{l} t^{3}-t+\varphi_{\infty}^{*}=0$. Assumptions $\varphi_{\infty}^{*} \neq 0, \alpha_{l}>\frac{4}{27} p_{s, 0}^{-1}\left|\varphi_{\infty}^{*}\right|^{-2}$ easily yields existence of a unique positive root $t_{0}=t_{0}^{+}$of said equation (when $\varphi_{\infty}^{*}<0$ ), and the same for the negative one, $t_{0}=t_{0}^{-}$(when $\varphi_{\infty}^{*}>0$ ). Conversely, let for instance $\varphi_{\infty}^{*}<0$ and take in (3.17) $t_{0}=t_{0}^{+}$. Function $\varphi(x)$ given now by formula (3.17) is bounded and continuous on $R^{1}$ (which is not difficult to be verified) and, letting $x \rightarrow+0$ (in (3.17)), we find $\varphi(0)=\varphi_{\infty}^{*}+p_{s, 0} \alpha_{l} t_{0}^{3}$; i.e. $\varphi(0)=t_{0}, \quad \beta_{l}\left[t_{0}\right]=\beta_{l}[\varphi(0)]=\beta_{l}[\varphi]$, and (3.17) shows that $\varphi(x)$ satisfies integral equation (3.16). Having the surface values $u(x, 0)=\varphi(x)$, it remains to solve the following two Dirichlet problems (as already noted in Sect.1, above): $\left\{\nabla^{2} u=0, z>0 ; u(x, 0)=\varphi(x), x \in R^{1}\right\} \quad$ and $\quad\left\{\nabla^{2} u=k_{b}^{-} u, z<0 ; u(x, 0)=\varphi(x), x \in R^{1}\right\} . \quad$ As it generally known, the relevant solutions are determined respectively by (3.18), (3.19).

Consider now the case of vacuum - vacuum heterogeneous system, with a semiconductor interface; then (3.14) is written as:

$$
\varphi-\varphi_{\infty}+\rho_{s}^{*}[\varphi]^{*} \psi_{s}^{0}=\beta_{l} \psi_{s}^{0}+\varepsilon_{s} \Delta \varphi_{\infty} \cdot \psi_{s}^{0,1}+\Delta \varphi_{\infty} \cdot \psi_{s}^{*}
$$

here $\beta_{l}$ is a given constant. Recall that $\rho_{s}^{*}[u] \equiv \omega_{d}^{0}\left(\varepsilon_{s} k_{s}^{2} \frac{\Delta \varphi_{\infty}}{2} s g-q_{s}[u]\right)$.

For equation (3.20) we shall establish existence of a unique continuous and bounded solution, via the contraction mapping argument. Let us introduce the notations $Q_{d}^{s}[\varphi](x)=\left(\left(\omega_{d}^{0} q_{s}[\varphi]\right) * \psi_{s}^{0}\right)(x)$ and

$$
f_{s}(x)=\Delta \varphi_{\infty}\left(\frac{s g(x)}{2}\left[1-\exp \left(-k_{s}|x|\right)\right]+\psi_{s}^{*}(x)-\frac{\varepsilon_{s} k_{s}^{2}}{2}\left(\left(\omega_{d}^{0} \cdot s g\right) * \psi_{s}^{0}\right)(x)+W_{L}\left[\psi_{s}^{0,1}\right](x)\right)+\beta_{l} \psi_{s}^{0}(x) \cdot(3 .
$$

Now, substituting $\psi_{s}^{0,1}$ in (3.20) with the right hand side of (3.15), equation (3.20) takes the form

$$
\varphi-\varphi_{\infty}^{*}-Q_{d}^{s}[\varphi]=f_{s}
$$

To analyze the above equation, we shall use the norm ||$w|| \equiv \sup _{x}|w(x)|, \quad x \in R^{1}$, for continuous, bounded functions $w(x)$ on $R^{1}$, and shall deal with balls $B_{r}\left(\varphi_{\infty}^{*}\right)$, centered at 
$\varphi_{\infty}^{*}$, having radius $r$; here $B_{r}\left(\varphi_{\infty}^{*}\right)$ is the closure, regarding the norm ||$.||$, to the set of the bounded continuous functions $v(x), x \in R^{1}$, such that ||$v-\varphi_{\infty}^{*}|| \leq r$, for a fixed $r$. It is clear that under the norm $\| .|| B_{r}\left(\varphi_{\infty}^{*}\right)$ is a complete metric space. We have to study the map $\varphi \rightarrow \Phi, \quad \Phi=\Phi[\varphi] \equiv \varphi_{\infty}^{*}+Q_{d}^{s}[\varphi]+f_{s}$, for $\varphi \in B_{r}\left(\varphi_{\infty}^{*}\right)$. For choosing a proper magnitude of radius $r$, we shall take into account that $Q_{d}^{s}\left[\varphi_{\infty}^{*}\right]=0$ and $f_{s}=\psi_{s}^{0}$ in case of the linear canonical form to problem (3.4), (3.5) (possessing $\psi_{s}^{0}$ as the unique solution). Thus, observing that ||$\psi_{s}^{0}||=\psi_{s}^{0}(0) \leq \frac{1}{2 \varepsilon_{s} k_{s}}$, we shall fix below a final choice of $r$ in the form $\frac{1+\Delta r}{2 \varepsilon_{s} k_{s}}$ ( $\Delta r$ - a small positive parameter). We begin the estimation to the image deviation $\Phi-\varphi_{\infty}^{*}, \Phi=\Phi[\varphi]$, by the obvious triangle inequality:

$$
|| \Phi-\varphi_{\infty}^{*}|| \leq|| Q_{d}^{s}[\varphi]||+|| f_{s}||,
$$

for $\varphi \in B_{r}\left(\varphi_{\infty}^{*}\right)$. By the inverse Fourier transformation of $\hat{\varphi}_{Q}(\xi) \quad\left(\varphi_{Q}=Q_{d}^{s}[\varphi]\right)$ we have $\left|Q_{d}^{s}[\varphi](x)\right| \leq|| q_{s}[\varphi]|| . \psi_{s}^{0}(0)$ (via the given definition of $Q_{d}^{s}[\varphi]$, formula (3.13) - for $\psi_{s}^{0}$ and equality $\left.\int_{-\infty}^{+\infty} \omega_{d}^{0}(x) d x=1\right)$, consequently it holds: ||$Q_{d}^{s}[\varphi]|| \leq|| q_{s}[\varphi]|| \frac{1}{2 \varepsilon_{s} k_{s}}$. Next, from ||$\varphi-\varphi_{\infty}^{*}|| \leq r$ we find for $q_{s}[\varphi]$ that ||$q_{s}[\varphi]|| \leq \frac{\left(\varepsilon_{s} k_{s}^{2} r\right)^{2}}{2}\left(1+\frac{\varepsilon_{s} k_{s}^{2} r}{3}\right)$. Choosing now $\Delta r=\frac{1}{2}$, we can fix the magnitude of $r$ :

$$
r=\frac{3}{4 \varepsilon_{s} k_{s}} .
$$

Then we find the next inequality, for ||$q_{s}[\varphi]||$ :

$$
|| q_{s}[\varphi]|| \leq \frac{9 k_{s}^{2}}{32}\left(1+\frac{3 k_{s}}{8}\right)
$$

Under condition $\frac{3}{4} \leq k_{s}^{-1}$ (applied below as a contraction requirement to $\Phi[\varphi]$ ) inequality (3.25) can be easily reworked till a convenient estimate for ||$q_{s}[\varphi]||$. We shall introduce, for a sake of simplicity, also a restriction in the form $k_{s}^{-1} \leq \kappa^{\prime}$ (with an arbitrary constant $\kappa^{\prime}>\frac{3}{4}$ ). For a large class of semiconductors (including these in [5], [7]) it is enough to take $\kappa^{\prime}=2$. Thus we can suppose from now on that

$$
\frac{3}{4} \leq k_{s}^{-1} \leq 2 \text {. }
$$


Now, reworking (3.25) we get: ||$q_{s}[\varphi]|| \leq \frac{3}{4}$. Consequently $Q_{d}^{s}[\varphi]$ is estimated as

$$
|| Q_{d}^{s}[\varphi]|| \leq \frac{r}{2}
$$

The obvious next step is to establish the analogous estimate for $f_{s}$, to get this way the needed property for $\Phi[\varphi]$ ( see (3.23)). For relevant terms with $\Delta \varphi_{\infty}$ in expression (3.21) it holds as follows. Function $\psi_{s}^{*}(x)$ satisfies (as directly shows (3.13)) inequality ||$\psi_{s}^{*}|| \leq \psi_{s}^{0}(0)$, therefore ||$\psi_{s}^{*}|| \leq \frac{1}{2 \varepsilon_{s} k_{s}}$. Next, estimating the term $\left(\omega_{d}^{0} \cdot s g\right) * \psi_{s}^{0}$ by analogy to $Q_{d}^{s}[\varphi]$, we have:

$$
||\left(\omega_{d}^{0} \cdot s g\right) * \psi_{s}^{0}|| \leq \frac{1}{2 \pi} \int_{-\infty}^{+\infty} \omega_{d}^{0}(x) d x \int_{-\infty}^{+\infty}\left|\hat{\psi}_{s}^{0}(\xi)\right| d \xi \leq \frac{1}{2 \varepsilon_{s} k_{s}}
$$

Concerning the element $W_{L}\left[\psi_{s}^{0,1}\right]$ (in said expression for $f_{s}$ ) we shall firstly introduce inequality

$$
|| W_{L}\left[\psi_{s}^{0,1}\right]|| \leq \frac{1}{2 \pi}|| 2|\xi|\left(k_{s}^{2}+\xi^{2}\right)^{-1}||_{L_{2}}|| \hat{\psi}_{s}^{0,1}||_{L_{2}}
$$

(where ||$.||_{L_{2}}$ is the norm in $L_{2}\left(R^{1}\right)$ ). Then we use that || $2|\xi|\left(k_{s}^{2}+\xi^{2}\right)^{-1}||_{L_{2}}=\left(\frac{2 \pi}{k_{s}}\right)^{1 / 2}$ and, because of (3.15), we have ||$\hat{\psi}_{s}^{0,1}||_{L_{2}} \leq|| \hat{\psi}_{s}^{0,1}-\frac{1}{\varepsilon_{s}}\left(W_{L}\left[\psi_{s}^{0,1}\right]\right)||_{L_{2}} \leq \frac{1}{2 \varepsilon_{s}}||\left(\operatorname{sg} \cdot \exp \left(-k_{s}|\cdot|\right)||_{L_{2}}\right.$ (with $(\Phi)$ as the Fourier transformation of $\Phi)$. The above yields inequality ||$W_{L}\left[\psi_{s}^{0,1}\right]|| \leq \frac{1}{\varepsilon_{s} k_{s} \sqrt{2}}<\frac{3}{4 \varepsilon_{s} k_{s}}$, taking into account that ||$\left(\operatorname{sg} \cdot \exp \left(-k_{s}||.\right)||_{L_{2}}=2\left(\frac{\pi}{k_{s}}\right)^{1 / 2} ;\right.$ i.e. ||$W_{L}\left[\psi_{s}^{0,1}\right]|| \leq r \quad$ (see (3.24)). Then, from inequality (see (3.21))

$$
|| f_{s}|| \leq\left|\Delta \varphi_{\infty}\right|\left(\frac{1}{2}+|| \psi_{s}^{*}||+\frac{\varepsilon_{s} k_{s}^{2}}{2}||\left(\omega_{d}^{0} \cdot s g\right) * \psi_{s}^{0}||+|| W_{L}\left[\psi_{s}^{0,1}\right]||\right)+\left|\beta_{l}\right||| \psi_{s}^{0}||,
$$

it follows the next one: ||$f_{s}|| \leq\left(\frac{1}{2}+\frac{2}{3} r+\frac{\varepsilon_{s} k_{s}^{2}}{4 \varepsilon_{s} k_{s}}+r\right)\left|\Delta \varphi_{\infty}\right|+\frac{2 r}{3}\left|\beta_{l}\right|$. Consequently, at $2 r \geq 1$, i.e. (via (3.24)) introducing condition (3.28), below, we find firstly that ||$f_{s}|| \leq r\left(\left[\frac{8}{3}+\frac{\varepsilon_{s} k_{s}^{2}}{3}\right]\left|\Delta \varphi_{\infty}\right|+\frac{2}{3}\left|\beta_{l}\right|\right)$. 


$$
\varepsilon_{s} k_{s} \leq \frac{3}{2}
$$

By (3.28) the above found for ||$f_{s}||$ modifies to inequality ||$f_{s}|| \leq \frac{r}{3}\left(14\left|\Delta \varphi_{\infty}\right|+2\left|\beta_{l}\right|\right)$. This will give the estimate

$$
|| f_{s}|| \leq \frac{r}{3}
$$

It holds when the sum $7\left|\Delta \varphi_{\infty}\right|+\left|\beta_{l}\right|$ satisfies condition

$$
7\left|\Delta \varphi_{\infty}\right|+\left|\beta_{l}\right| \leq \frac{1}{2}
$$

Summarizing (3.23), (3.27) and (3.29), we conclude the following. For any data $\varphi_{\infty}^{+}, \varphi_{\infty}^{-}, \beta_{l}$ and parameters $k_{s}, \varepsilon_{s}$ satisfying (3.26), (3.28) and (3.30) relation $\Phi=\Phi[\varphi]$ maps the ball $B_{r}\left(\varphi_{\infty}^{*}\right)$, with $r$ as in (3.24), into itself.

Now we shall study the contraction property of $\Phi[\varphi]$. For arbitrary two elements $\varphi_{1}, \varphi_{2} \in B_{r}\left(\varphi_{\infty}^{*}\right)$ we have to estimate difference $\Phi_{2}-\Phi_{1}, \Phi_{j}=\Phi\left[\varphi_{j}\right] \quad(j=1,2)$. From $\Phi_{2}-\Phi_{1}=Q_{d}^{s}\left[\varphi_{2}\right]-Q_{d}^{s}\left[\varphi_{1}\right]$ we shall consider difference of $Q_{d}^{s}\left[\varphi_{j}\right](j=1,2)$, using relation

$$
Q_{d}^{s}\left[\varphi_{2}\right]-Q_{d}^{s}\left[\varphi_{1}\right]=\left(\omega_{d}^{0}\left(\varphi_{2}-\varphi_{1}\right) \int_{0}^{1} q_{s}^{\prime}\left[\varphi_{1}+\tau\left(\varphi_{2}-\varphi_{1}\right)\right] d \tau\right) * \psi_{s}^{0}
$$

(Above $q_{s}^{\prime}[t]$ is derivative $\frac{d q_{s}}{d t}[t]$, with $q_{s}[t] \equiv \frac{1}{2 !}\left[\varepsilon_{s} k_{s}^{2}\left(t-\varphi_{\infty}^{*}\right)\right]^{2}-\frac{1}{3 !}\left[\varepsilon_{s} k_{s}^{2}\left(t-\varphi_{\infty}^{*}\right)\right]^{3}$.) Denote by $R_{d}^{s}\left[\varphi_{2,1}\right] * \psi_{s}^{0}$ the right hand side of (3.31) and take into account the next several inequalities:

$$
\begin{gathered}
|| Q_{d}^{s}\left[\varphi_{2}\right]-Q_{d}^{s}\left[\varphi_{1}\right]|| \leq \frac{1}{2 \pi} \int_{-\infty}^{+\infty}\left|\hat{R}_{d}^{s}\left[\varphi_{2,1}\right](\xi)\right| \cdot\left|\hat{\psi}_{s}^{0}(\xi)\right| d \xi ; \\
|| \hat{R}_{d}^{s}\left[\varphi_{2,1}\right]|| \leq|| \varphi_{2}-\varphi_{1}|| \int_{-\infty}^{+\infty} \omega_{d}^{0}(x) \int_{0}^{1}\left|q_{s}^{\prime}\left[\varphi_{1}+\tau\left(\varphi_{2}-\varphi_{1}\right)\right](x)\right| d \tau d x ; \\
|| q_{s}^{\prime}[\cdot]|| \leq \varepsilon_{s} k_{s}^{2} r\left(1+\frac{\varepsilon_{s} k_{s}^{2} r}{2}\right)\left(\text { at } \varphi_{1}, \varphi_{2} \in B_{r}\left(\varphi_{\infty}^{*}\right)\right), \text { i.e. }|| q_{s}^{\prime}[\cdot]|| \leq \frac{3 k_{s}}{4}\left(1+\frac{3 k_{s}}{8}\right) \leq \frac{3}{2} ; \\
\int_{0}^{1}|| q_{s}^{\prime}\left[\varphi_{1}+\tau\left(\varphi_{2}-\varphi_{1}\right)\right]|| d \tau \leq \frac{3}{2} .
\end{gathered}
$$

(We have used in (3.32) relation (3.26), via (3.24), and the above notations $\hat{\psi}_{s}^{0}, \hat{R}_{d}^{s}\left[\varphi_{2,1}\right]$ are taken for the Fourier images respectively to functions $\psi_{s}^{0}(x), R_{d}^{s}\left[\varphi_{2,1}\right](x)$.) Then we have: 


$$
|| Q_{d}^{s}\left[\varphi_{2}\right]-Q_{d}^{s}\left[\varphi_{1}\right]|| \leq \frac{3}{2}|| \varphi_{2}-\varphi_{1}|| \frac{1}{2 \pi} \int_{-\infty}^{+\infty}\left|\hat{\psi}_{s}^{0}(\xi)\right| d \xi \leq \frac{3}{4 \varepsilon_{s} k_{s}}|| \varphi_{2}-\varphi_{1}||
$$

Consequently,

$$
|| \Phi_{2}-\Phi_{1}|| \leq r|| \varphi_{2}-\varphi_{1}||
$$

(because of (3.24)), and under estimates (3.27), (3.29) (valid at (3.30)) the considered map $\Phi=\Phi[\varphi]$ is a contraction in the ball $B_{r}\left(\varphi_{\infty}^{*}\right)$, for $r<1$, i.e. $\frac{3}{4}<\varepsilon_{s} k_{s}$. Combining the latter inequality with (3.28), we come to condition

$$
\frac{3}{4}<\varepsilon_{s} k_{s} \leq \frac{3}{2} .
$$

Thus we have given the proof of the following basic result.

\subsection{Proposition}

For arbitrary values of positive parameters $\varepsilon_{s}, k_{s}$, each asymptotic data $\varphi_{\infty}^{ \pm}$of the surface potential and line charges $\rho_{l}=\varepsilon_{0} \beta_{l}$, such that conditions (3.26), (3.33) and (3.30) hold, equation (3.20) possesses a unique continuous, bounded solution $\varphi(x ; d)$, satisfying the estimate

$$
\sup _{x}\left|\varphi(x ; d)-\varphi_{\infty}^{*}\right| \leq \frac{5}{8 \varepsilon_{s} k_{s}}, \forall d>0
$$

\section{Explicit approximations in the case of semiconductor interface}

Via the possible applications, it is important to ask for a suitable approximation $\varphi_{*}^{0}(x)$ to the interface data $u(x, 0)$, well enough at small $|d|$ and explicitly determined. To that goal, suppose a sequence $\left\{\varphi_{n}\right\}$ of solutions to $(3.20), \varphi_{n}(x) \equiv \varphi(x ; d)$, at $d=d_{n}$, with $d_{n} \rightarrow 0$ $(n \rightarrow \infty)$, is convergent (in a distribution sense) to a bounded continuous function $\varphi_{*}(x)$, $x \in R^{1}$. Putting $d=d_{n}$ in (3.20) and letting $n \rightarrow \infty$, we can conclude that $\varphi_{*}(x)$ is a solution to equation

$$
\varphi(x)=\varphi_{\infty}+\left(\beta_{l}+q_{s}[\varphi(0)]\right) \psi_{s}^{0}(x)+\Delta \varphi_{\infty}\left(\psi_{s}^{*}(x)+\varepsilon_{s} \psi_{s}^{0,1}(x)\right) .
$$

For finding (4.1) we have taken into account that $Q_{d}^{s}[\varphi(. ; d)](x) \rightarrow q_{s}\left[\varphi_{*}(0)\right] \psi_{s}^{0}(x)$ and $\left[\left(\omega_{d}^{0} \cdot s g\right)^{*} \psi_{s}^{0}\right](x) \rightarrow 0, \forall x \in R^{1}$, at $d=d_{n}(n \rightarrow \infty)$. Next we shall study equation (4.1). Note first of all the necessary condition to have a continuous solution $\varphi(x)$ :

$$
\varphi_{\infty}^{-}+\varepsilon_{s} \Delta \varphi_{\infty} \psi_{s}^{0,1}(-0)=\varphi_{\infty}^{+}+\varepsilon_{s} \Delta \varphi_{\infty} \psi_{s}^{0,1}(+0) ;
$$

it is fulfilled, because of (3.15). If $\varphi(x)$ is a continuous solution to (4.1), for value $\varphi(0)$ we obtain (from (4.1), at $x=+0$ or $x=-0$ ) the algebraic equation 


$$
\varphi(0)=\varphi_{\infty}^{*}+\left(\beta_{l}+q_{s}[\varphi(0)]\right) \psi_{s}^{0}(0)
$$

i.e. $\varphi(0)$ is a real solution to equation

$$
\psi_{s}^{0}(0) \frac{\left(\varepsilon_{s} k_{s}^{2}\right)^{3}}{6}\left(z-\varphi_{\infty}^{*}\right)^{3}-\psi_{s}^{0}(0) \frac{\left(\varepsilon_{s} k_{s}^{2}\right)^{2}}{2}\left(z-\varphi_{\infty}^{*}\right)^{2}+z-\varphi_{\infty}^{*}-\beta_{l} \psi_{s}^{0}(0)=0 \text {. }
$$

Setting $t=\varepsilon_{s} k_{s}^{2}\left(z-\varphi_{\infty}^{*}\right)$ we rewrite this equation in the form:

$$
\frac{t^{3}}{6}-\frac{t^{2}}{2}+\frac{t}{\varepsilon_{s} k_{s}^{2} \psi_{s}^{0}(0)}-\beta_{l}=0 .
$$

Derivative of the left hand side (denote it by $g(t))$ is $g^{\prime}(t)=\frac{t^{2}}{2}-t+\frac{1}{\varepsilon_{s} k_{s}^{2} \psi_{s}^{0}(0)}$. The found quadratic polynomial does not have real roots (via (3.26) and the known inequality $\psi_{s}^{0}(0) \leq \frac{1}{2 \varepsilon_{s} k_{s}}$, recall $(3.13)$ concerning $\left.\psi_{s}^{0}(0)\right)$. This yields existence of a unique real solution $t^{0}$ of (4.2) and we set

$$
\varphi^{0}=\varphi_{\infty}^{*}+\frac{t^{0}}{\varepsilon_{s} k_{s}^{2}} .
$$

Now from (4.1) we get the function

$$
\varphi_{*}^{0}(x) \equiv \varphi_{\infty}+\left(\beta_{l}+q_{s}\left[\varphi^{0}\right]\right) \psi_{s}^{0}(x)+\Delta \varphi_{\infty}\left(\psi_{s}^{*}(x)+\varepsilon_{s} \psi_{s}^{0,1}(x)\right) .
$$

It presents actually the unique solution of equation (4.1).

The next step will be the comparison of $\varphi_{*}^{0}(x)$ and $\varphi(x ; d)$. Let us introduce the difference $\left.\Delta Q_{d}^{s}[\varphi](x) \equiv Q_{d}^{s}[\varphi](x)-q_{s}[\varphi(0)]\right) \psi_{s}^{0}(x)$. Formula (4.4) then directly shows that $\varphi_{*}^{0}(x)$ is a solution to equation

$$
\varphi(x)-\varphi_{\infty}^{*}-Q_{d}^{s}[\varphi](x)=f_{s}(x)-\Delta Q_{d}^{s}[\varphi](x)
$$

Subtracting (4.5), with $\varphi=\varphi_{*}^{0}$, from (3.20) - with $\varphi=\varphi(. ; d)$, we evidently get:

$$
\varphi(. ; d)-\varphi_{*}^{0}=Q_{d}^{s}[\varphi(. ; d)]-Q_{d}^{s}\left[\varphi_{*}^{0}\right]+\Delta Q_{d}^{s}\left[\varphi_{*}^{0}\right] .
$$

Putting afterwards $\varphi_{2}=\varphi(. ; d)$ and $\varphi_{1}=\varphi_{*}^{0}$ in the above given contraction estimate - for $Q_{d}^{s}\left[\varphi_{2}\right]-Q_{d}^{s}\left[\varphi_{1}\right]$, we find directly that

$$
|| \varphi(. ; d)-\varphi_{*}^{0}|| \leq r|| \varphi(. ; d)-\varphi_{*}^{0}||+|| \Delta Q_{d}^{s}\left[\varphi_{*}^{0}\right]||,
$$

consequently

$$
|| \varphi(. ; d)-\varphi_{*}^{0}|| \leq \frac{1}{1-r}|| \Delta Q_{d}^{s}\left[\varphi_{*}^{0}\right]||
$$


By the known definition $Q_{d}^{s}[\varphi] \equiv\left(\omega_{d}^{0} q_{s}[\varphi]\right) * \psi_{s}^{0}$, perturbation term $\Delta Q_{d}^{s}\left[\varphi_{*}^{0}\right]$ can be easily presented as

$$
\begin{aligned}
& \Delta Q_{d}^{s}\left[\varphi_{*}^{0}\right](x)=\int_{-\infty}^{+\infty} \omega^{0}(\tau)\left(q_{s}\left[\varphi_{*}^{0}(\tau d)\right]-q_{s}\left[\varphi^{0}\right]\right) \psi_{s}^{0}(x-\tau d) d \tau+ \\
& +q_{s}\left[\varphi^{0}\right] \int_{-\infty}^{+\infty} \omega^{0}(\tau)\left[\psi_{s}^{0}(x-\tau d)-\psi_{s}^{0}(x)\right] d \tau
\end{aligned}
$$

Denote the first and second integrals in (4.7) respectively by $I_{1}\left[\varphi_{*}^{0}\right](x)$ and $I_{2}\left[\varphi_{*}^{0}\right](x)$; they satisfy the following inequalities:

$$
\begin{gathered}
|| I_{1}\left[\varphi_{*}^{0}\right]|| \leq 2|| \psi_{s}^{0}|| \sup _{|y| \leq d}\left|q_{s}\left[\varphi_{*}^{0}(y)\right]-q_{s}\left[\varphi^{0}\right]\right| ; \\
|| I_{2}\left[\varphi_{*}^{0}\right]|| \leq\left|q_{s}\left[\varphi^{0}\right]\right| \int_{-\infty}^{+\infty} \omega^{0}(\tau) \sup _{x}\left|\psi_{s}^{0}(x-\tau d)-\psi_{s}^{0}(x)\right| d \tau .
\end{gathered}
$$

To rework estimate (4.8.a), taking the arguments, known from the analysis of (3.29) (see above), we use at the beginning that

$$
|| q_{s}\left[\varphi_{*}^{0}\right]-q_{s}\left[\varphi^{0}\right]||_{d} \leq \varepsilon_{s} k_{s}^{2}\left(1+\frac{\varepsilon_{s} k_{s}^{2}}{2}\right)|| \varphi_{*}^{0}-\varphi^{0}||_{d},
$$

where

$$
\begin{gathered}
|| \varphi_{*}^{0}-\varphi^{0}||_{d} \equiv \sup _{|y| \leq d}\left|\varphi_{*}^{0}(y)-\varphi^{0}\right| ; \text { i.e. } \\
|| q_{s}\left[\varphi_{*}^{0}\right]-q_{s}\left[\varphi^{0}\right]||_{d} \leq \frac{3 k_{s}}{2}\left(1+\frac{3}{4} k_{s}\right)|| \varphi_{*}^{0}-\varphi^{0}||_{d} \leq 4|| \varphi_{*}^{0}-\varphi^{0}||_{d},
\end{gathered}
$$

via (3.26), (3.33). Substituting then in (4.8.a), we have:

$$
|| I_{1}\left[\varphi_{*}^{0}\right]|| \leq 8|| \psi_{s}^{0}|| .|| \varphi_{*}^{0}-\varphi^{0}||_{d} .
$$

(We shall show afterwards that ||$\varphi_{*}^{0}-\varphi^{0}||_{d} \leq 1$ at small enough $d$.) Next, for reworking of (4.8.b), we shall firstly apply equality

$$
\psi_{s}^{0}(x-y)-\psi_{s}^{0}(x)=-y \int_{0}^{1} \psi_{s}^{0,1}(x-t y) d t .
$$

(Via formula (3.13) for $\psi_{s}^{0}(x)$ it is not difficult to verify validity of the above.) Consequently $\sup _{x}\left|\psi_{s}^{0}(x-y)-\psi_{s}^{0}(x)\right| \leq|y| .|| \psi_{s}^{0,1}||$, and, because of (4.8.b), we find inequality

$$
|| I_{2}\left[\varphi_{*}^{0}\right]|| \leq\left|q_{s}\left[\varphi^{0}\right]\right| \cdot|| \psi_{s}^{0,1}|| d \int_{-\infty}^{+\infty}|\tau| \omega^{0}(\tau) d \tau,
$$

which actually yields that: 


$$
|| I_{2}\left[\varphi_{*}^{0}\right]|| \leq\left|q_{s}\left[\varphi^{0}\right]\right| \cdot|| \psi_{s}^{0,1}|| d .
$$

To final reworking of (4.8.a) we shall estimate the quantity ||$\varphi_{*}^{0}-\varphi^{0}||_{d}$ in (4.9.a). By (4.4) and the algebraic equation $\varphi(0)=\varphi_{\infty}^{*}+\left(\beta_{l}+q_{s}[\varphi(0)]\right) \psi_{s}^{0}(0)$ for $\varphi(0)$ (see the initial form of (4.2)) we firstly have

$$
\varphi_{*}^{0}(x)-\varphi^{0}=\frac{\Delta \varphi_{\infty}}{2} s g(x)+\left(\beta_{l}+q_{s}\left[\varphi^{0}\right]\right)\left[\psi_{s}^{0}(x)-\psi_{s}^{0}(0)\right]+\Delta \varphi_{\infty}\left[\psi_{s}^{*}(x)+\varepsilon_{s} \psi_{s}^{0,1}(x)\right],
$$

and expressing $\psi_{s}^{0,1}$ by (3.15) we get

$$
\begin{gathered}
\varphi_{*}^{0}(x)-\varphi^{0}=\frac{\Delta \varphi_{\infty}}{2} s g(x)\left[1-\exp \left(k_{s}|x|\right)\right]+\left(\beta_{l}+q_{s}\left[\varphi^{0}\right]\right)\left[\psi_{s}^{0}(x)-\psi_{s}^{0}(0)\right]+ \\
+\Delta \varphi_{\infty}\left(\psi_{s}^{*}(x)+W_{L}\left[\psi_{s}^{0,1}\right](x)\right) .
\end{gathered}
$$

Next we analyze the terms in (4.10) beginning with function $W_{L}\left[\psi_{S}^{0,1}\right](x)$; it is continuous and odd, and we can use the following inequalities:

$$
\left|W_{L}\left[\psi_{s}^{0,1}\right]\right|(x) \leq \frac{2}{\pi} \int_{0}^{+\infty} \frac{\xi|\sin (x \xi)|}{k_{s}^{2}+\xi^{2}}\left|\hat{\psi}_{s}^{0,1}(\xi)\right| d \xi \leq \frac{1}{\pi}\left(\int_{0}^{+\infty} \frac{\xi^{2}\left|\sin ^{2}(x \xi)\right|}{\left(k_{s}^{2}+\xi^{2}\right)^{2}} d \xi\right)^{1 / 2} \cdot|| \hat{\psi}_{s}^{0,1}||_{L_{2}\left(R^{1}\right)} .
$$

Therefore:

$$
\left|W_{L}\left[\psi_{s}^{0,1}\right]\right|(x) \leq \frac{\sqrt{|x|}}{\pi}\left(\int_{0}^{+\infty} \frac{\sin ^{2} \theta}{\theta^{2}} d \theta\right)^{1 / 2} \cdot|| \hat{\psi}_{s}^{0,1}||_{L_{2}\left(R^{1}\right)} .
$$

Recall here the already found estimate ||$\hat{\psi}_{s}^{0,1}||_{L_{2}\left(R^{1}\right)} \leq \frac{\sqrt{\pi}}{\varepsilon_{s} \sqrt{k_{s}}}$ and the well known relation $\int_{0}^{+\infty} \frac{\sin ^{2} \theta}{\theta^{2}} d \theta=\frac{\pi}{2}$. Then it follows:

$$
|| W_{L}\left[\psi_{s}^{0,1}\right]||_{d} \leq \frac{\sqrt{d}}{\varepsilon_{s} \sqrt{k_{s}}}
$$

For $\psi_{s}^{*}(x)$ we start with the inequality

$$
\left|\psi_{s}^{*}(x)\right| \leq \frac{|x|}{\pi \varepsilon_{s}} \int_{0}^{1 / d}\left|\frac{\sin (x \xi)}{x \xi}\right| \frac{\xi}{k_{s}^{2}+\xi^{2}} d \xi+\frac{1}{\pi \varepsilon_{s}} \int_{1 / d}^{\infty} \frac{|\sin (x \xi)|}{k_{s}^{2}+\xi^{2}} d \xi
$$

i.e.:

$$
\left|\psi_{s}^{*}(x)\right| \leq \frac{|x|}{2 \pi \varepsilon_{s}} \log \left(1+\frac{1}{k_{s}^{2} d^{2}}\right)+\frac{1}{\pi \varepsilon_{s}} \int_{1 / d}^{\infty} \frac{1}{\xi^{2}} d \xi \leq \frac{|x|}{\pi \varepsilon_{s}} \log \left(1+\frac{1}{k_{s} d}\right)+\frac{d}{\pi \varepsilon_{s}} .
$$


Evidently, $\log \left(1+\frac{1}{k_{s} d}\right) \leq \frac{1}{\sqrt{k_{s} d}}$, and we find:

$$
|| \psi_{s}^{*}||_{d} \leq \frac{1}{\pi \varepsilon_{s}}\left(d+\frac{\sqrt{d}}{\sqrt{k_{s}}}\right) .
$$

The estimate for $\psi_{s}^{0}(x)-\psi_{s}^{0}(0)$ is a consequence from the above one for $\psi_{s}^{0}(x-y)-\psi_{s}^{0}(x)$, thus we come to inequality

$$
|| \psi_{s}^{0}-\psi_{s}^{0}(0)||_{d} \leq d|| \psi_{s}^{0,1}||,
$$

and for $1-\exp \left(k_{s}|x|\right)$ we can take the next obviously one:

$$
|| 1-\exp \left(k_{s}|x|\right)||_{d} \leq k_{s} d \exp \left(k_{s} d\right) .
$$

Now (4.10) and (4.11) - (4.14) yield:

$$
|| \varphi_{*}^{0}-\varphi^{0}||_{d} \leq\left|\Delta \varphi_{\infty}\right|\left(\frac{\sqrt{k_{s} d}}{2} \exp \left(k_{s} d\right)+\frac{4 d \sqrt{d}}{9 \varepsilon_{s}^{2} \sqrt{k_{s}}}\right)+\left(\left|\beta_{l}\right|+\left|q_{s}\left[\varphi^{0}\right]\right|\right)|| \psi_{s}^{0,1}|| d .
$$

The above simplifies, at $d k_{s} \leq 1$, to inequality

$$
|| \varphi_{*}^{0}-\varphi^{0}||_{d} \leq\left|\Delta \varphi_{\infty}\right|\left(\frac{3}{2}+\frac{20}{9} r\right) \sqrt{d k_{s}}+\left(\left|\beta_{l}\right|+\left|q_{s}\left[\varphi^{0}\right]\right|\right) \frac{3 d}{4 \varepsilon_{s}}\left(1+\frac{1}{\varepsilon_{s} k_{s}}\right)
$$

i.e.

$$
|| \varphi_{*}^{0}-\varphi^{0}||_{d} \leq\left[6\left|\Delta \varphi_{\infty}\right|+\left(1+\frac{4}{3}\right)\left(\left|\beta_{l}\right|+\left|q_{s}\left[\varphi^{0}\right]\right|\right)\right] r \sqrt{d k_{s}},
$$

and

$$
|| \varphi_{*}^{0}-\varphi^{0}||_{d} \leq 3\left(2\left|\Delta \varphi_{\infty}\right|+\left|\beta_{l}\right|+\left|q_{s}\left[\varphi^{0}\right]\right|\right) \sqrt{d k_{s}} .
$$

Going to expression (4.7), we have firstly ||$\Delta Q_{d}^{s}\left[\varphi_{*}^{0}\right]|| \leq|| I_{1}\left[\varphi_{*}^{0}\right]||+|| I_{2}\left[\varphi_{*}^{0}\right]||$ and applying afterwards (4.9.a), combined with (4.15) and (4.9.b), we establish the estimate:

$$
|| \Delta Q_{d}^{s}\left[\varphi_{*}^{0}\right]|| \leq 8|| \psi_{s}^{0}|| .|| \varphi_{*}^{0}-\varphi^{0}||_{d}+\left|q_{s}\left[\varphi^{0}\right]\right| .|| \psi_{s}^{0,1}|| d .
$$

Next, for the relevant quantities in (4.16) it can be easily established (via (3.26), (3.33)) as follows:

$$
\begin{gathered}
8|| \psi_{s}^{0}|| .|| \varphi_{*}^{0}-\varphi^{0}||_{d} \leq \frac{12}{\varepsilon_{s} k_{s}}\left(2\left|\Delta \varphi_{\infty}\right|+\left|\beta_{l}\right|+\left|q_{s}\left[\varphi^{0}\right]\right|\right) \sqrt{d k_{s}} \leq 16\left(2\left|\Delta \varphi_{\infty}\right|+\left|\beta_{l}\right|+\left|q_{s}\left[\varphi^{0}\right]\right|\right) \sqrt{d k_{s}} \\
\left|q_{s}\left[\varphi^{0}\right]\right| .|| \psi_{s}^{0,1}|| d \leq\left(1+\frac{4}{3}\right)\left|q_{s}\left[\varphi^{0}\right]\right| \frac{3 d}{4 \varepsilon_{s}} \leq 3\left|q_{s}\left[\varphi^{0}\right]\right| \sqrt{d k_{s}} .
\end{gathered}
$$


Applying the above two inequalities to (4.16), we find that

$$
|| \Delta Q_{d}^{s}\left[\varphi_{*}^{0}\right]|| \leq 8\left(4\left|\Delta \varphi_{\infty}\right|+2\left|\beta_{l}\right|+3\left|q_{s}\left[\varphi^{0}\right]\right|\right) \sqrt{d k_{s}} .
$$

Finally, from (4.6), (4.17) we directly get:

$$
|| \varphi(. ; d)-\varphi_{*}^{0}|| \leq \frac{8}{1-r}\left(4\left|\Delta \varphi_{\infty}\right|+3\left|q_{s}\left[\varphi^{0}\right]\right|+2\left|\beta_{l}\right|\right) \sqrt{d k_{s}} .
$$

Now let us introduce (for a simplicity sake) the restriction $0.5 \leq r \leq 0.9$, equivalent to condition

$$
\frac{5}{6} \leq \varepsilon_{s} k_{s} \leq \frac{3}{2}
$$

Then $1-r \geq 0.1$, consequently $\frac{8}{1-r} \leq 80$, and, from the above inequality for $\varphi(. ; d)-\varphi_{*}^{0}$, we obtain the needed estimate, approximating for the exact solution $\varphi(x ; d)$, at $d \rightarrow 0$ :

$$
|| \varphi(. ; d)-\varphi_{*}^{0}|| \leq 80\left(2\left|\beta_{l}\right|+3\left|q_{s}\left[\varphi^{0}\right]\right|+4\left|\Delta \varphi_{\infty}\right|\right) \sqrt{d k_{s}} .
$$

By the above arguments we have actually proven the following assertion:

\subsection{Proposition}

Function $\varphi_{*}^{0}(x)$ is an approximation to solution $\varphi(x ; d)$ of equation (3.20), at $d \rightarrow 0$, explicitly determined by formula (4.4) and satisfying estimate (4.19), for parameters $\varphi_{\infty}^{ \pm}, \beta_{l}$, which fulfill (3.30) and $\left(\varepsilon_{s}, k_{s}\right)$ varying in the compact set determined by (3.26), (4.18).

\section{Concluding remarks}

Here we accent on the approximating solutions, in several applicable variants, via the convenience of solution determination by effective formulas (see positions 2) - 5), below). Note, as a principle, that the possible explicit solutions (presenting for instance the interface electric potential) are necessary for examination of relevant numerical methods, and the same holds for the explicit approximations to the exact implicit solutions. Below we start with some dimensional remarks, related in particular to known experimental data.

1. In a $\left(k_{s}^{-1}, \varepsilon_{s}\right)$ - coordinate system, scaled in nanometers, the above mentioned compact is trapezoid, with contours - the straight lines: $k_{s}^{-1}=\frac{3}{4} n m$ and $k_{s}^{-1}=2 n m$ (as the bottoms of trapezoid, vertically situated), and $\varepsilon_{s}=\frac{5}{6} k_{s}^{-1} n m, \varepsilon_{s}=\frac{3}{2} k_{s}^{-1} n m$ (as the thighs). For the classes of semiconductors analyzed in [5], [7] the values of parameter $k_{s}^{-1}$ are not greater than $1 \mathrm{~nm}$, satisfying thus condition (3.26) in the form

$$
0.75 \mathrm{~nm} \leq k_{s}^{-1} \leq 2 \mathrm{~nm} .
$$


The key non-dimensional quantity in the surface electrostatics is given by the product $\varepsilon_{s} k_{s}$, and the same holds for the above used $d k_{s}$. On the other hand, quantity $\left(\varepsilon_{s} k_{s}\right)^{-1}$ can be automatically provided with a (preferable) voltage dimension (see also expression (3.24) of $r)$. Recall that such a mechanism has been suggested by the estimate ||$\psi_{s}^{0}||=\psi_{s}^{0}(0) \leq \frac{1}{2 \varepsilon_{s} k_{s}}$ of canonical surface potential $\psi_{s}^{0}$. This allows, for mathematical reworks, to use the product $k_{s} r$ (in the important factor $\varepsilon_{s} k_{s}^{2} r=\varepsilon_{s} k_{s} \cdot k_{s} r$ ) as nondimensional.

2. The proposed model (2.2) - (2.6), with $\varepsilon_{b}^{-}=1, k_{b}^{-}=0$, admits explicit approximations $\varphi_{*}^{0}(x)$ and $u_{*}^{0}(x, z)$,

$$
\begin{gathered}
\varphi(x ; d)=\varphi_{*}^{0}(x)+O\left(\sqrt{d k_{s}}\right)(d \rightarrow 0), \forall x \in R^{1} ; \\
u(x, z)=u_{*}^{0}(x, z)+O\left(\sqrt{d k_{s}}\right)(d \rightarrow 0), \forall(x, z) \in R^{2} .
\end{gathered}
$$

They satisfy estimates (4.19) and

$$
\sup _{z}|| u(., z)-u_{*}^{0}(., z)|| \leq 80\left(2\left|\beta_{l}\right|+3\left|q_{s}\left[\varphi^{0}\right]\right|+4\left|\Delta \varphi_{\infty}\right|\right) \sqrt{d k_{s}} .
$$

In addition, said approximations are determined respectively by formulas (4.4) and

$$
u_{*}^{0}(x, z)=\left\{\begin{array}{c}
\frac{|z|}{\pi} \int_{-\infty}^{+\infty} \frac{\varphi_{*}^{0}(t)}{(x-t)^{2}+z^{2}} d t, z \neq 0 \\
\varphi_{*}^{0}(x), \quad z=0
\end{array} .\right.
$$

3. In case of relatively small $\Delta \varphi_{\infty}$, i.e. $\left|\Delta \varphi_{\infty}\right|<<\left|\varphi_{\infty}^{*}\right|$, the term $\Delta \varphi_{\infty}\left(\psi_{s}^{*}(x)+\varepsilon_{s} \psi_{s}^{0,1}(x)\right)$ can be neglected in representation (4.4) and we can use the simplified approximation $\varphi_{0, *}(x) \equiv \varphi_{\infty}+\left(\beta_{l}+q_{s}\left[\varphi^{0}\right]\right) \psi_{s}^{0}(x)$ of $\varphi(x ; d)$, instead of $\varphi_{*}^{0}(x)$. This yields the simpler approximation $u_{0, *}(x, z)$ to the space potential $u(x, z)$, with

$$
u_{0, *}(x, z)=\varphi_{\infty}(x)+\frac{\beta_{l, s}}{\pi} \int_{0}^{+\infty} \frac{\exp (-|z| \xi) \cos (x \xi)}{2 \xi+\varepsilon_{s}\left(k_{s}^{2}+\xi^{2}\right)} d \xi, z \in R^{1}
$$

Above $\beta_{l, s}=\beta_{l}+q_{s}\left[\varphi^{0}\right]$. At $z=0$ formula (4.23) evidently gives $u_{0, *}(x, 0)=\varphi_{0, *}(x)$. Here it should be specially noted that known real situations (see for instance in [5], [7]) are contained in the case $\Delta \varphi_{\infty}=0$.

4. The case $\varphi_{\infty}^{-}=\varphi_{\infty}^{+}=0$ (then $\Delta \varphi_{\infty}=0$ and $\varphi_{\infty}^{*}=0$ ) covers the experimental models in [5], [7]. Now it seems to be an open question whether the line phase charges can get essentially smaller values than these for the surface zones (called terraces) $\{z=0, d<<|x|\}$ - after specific annealing of indium-phosphorus semiconductor 
samples, say ([5], [7]). Then we would have relatively small values of $\left|\beta_{l}\right|$, terms with $t^{2}$ and $t^{3}\left(\right.$ at $t=t^{0}$ ) can be neglected in (4.2), and we can take the value of $p_{s, 0} \beta_{l}$ (with $\left.p_{s, 0}=\psi_{s}^{0}(0)\right)$ as an approximation of $\frac{t^{0}}{\varepsilon_{s} k_{s}^{2}}$ (using (4.2)). It gives that $q_{s}\left[\varphi^{0}\right] \approx \frac{1}{2} \varepsilon_{s}^{2} k_{s}^{4} p_{s, 0}^{2} \beta_{l}^{2}$. Replace in (4.23) $\beta_{l, s} \quad$ with $\beta_{l}+\frac{1}{2} \varepsilon_{s}^{2} k_{s}^{4} p_{s, 0}^{2} \beta_{l}^{2}$, and take $\psi_{s}^{0}(0) \approx \frac{1}{2 \varepsilon_{s} k_{s}}$ (via the known estimate $\left.\psi_{s}^{0}(0) \leq \frac{1}{2 \varepsilon_{s} k_{s}}\right)$. Thus we can consider the function $w_{0, *}(x, z)$, below, as a next approximation of the exact potential $u(x, z)$.

$$
w_{0, *}(x, z)=\frac{\beta_{l}+8^{-1} \cdot k_{s}^{2} \beta_{l}^{2}}{\pi} \int_{0}^{+\infty} \frac{\exp (-|z| \xi) \cos (x \xi)}{2 \xi+\varepsilon_{s}\left(k_{s}^{2}+\xi^{2}\right)} d \xi, z \in R^{1} .
$$

The found formula conveniently shows that the (nonlinear) impact of the vacancy denuded sub-strips $\{z=0, \theta d<|x|<d\} \quad$ (with $0<\theta<1$ ) is compatible to the perturbation $\frac{k_{s}^{2} \beta_{l}^{2}}{8 \pi} \int_{0}^{+\infty} \frac{\exp (-|z| \xi) \cos (x \xi)}{2 \xi+\varepsilon_{s}\left(k_{s}^{2}+\xi^{2}\right)} d \xi, z \in R^{1}$.

5. A special variant is presented by the case of weakly charged contour $\{z=0, x=0\}$, combined with a relatively higher asymptotic surface power $\Delta \varphi_{\infty}$. From experimental view point (c.f. [5], [7]) said situation seems to be another open question. Now we can assume that $\left|\beta_{l}\right|<<\left|\Delta \varphi_{\infty}\right|$. Then neglecting term $\left(\beta_{l}+q_{s}\left[\varphi^{0}\right]\right) \psi_{s}^{0}(x)$ in the expression for $\varphi_{*}^{0}(x)$, we insert in (4.22) $\varphi_{\infty}(t)+\Delta \varphi_{\infty}\left(\psi_{s}^{*}(t)+\varepsilon_{s} \psi_{s}^{0,1}(t)\right)$ (instead of $\left.\varphi_{*}^{0}(t)\right)$ and find the following expression:

$$
w_{0, \infty}(x, z)=\varphi_{\infty}(x)-\frac{\Delta \varphi_{\infty}}{\pi} \int_{0}^{+\infty} \frac{\exp (-|z| \xi)\left(1+\varepsilon_{s} \xi\right) \sin (x \xi)}{2 \xi+\varepsilon_{s}\left(k_{s}^{2}+\xi^{2}\right)} d \xi, z \neq 0
$$

Here $w_{0, \infty}(x, 0)=\varphi_{\infty}(x)+\Delta \varphi_{\infty}\left(\psi_{s}^{*}(x)+\varepsilon_{s} \psi_{s}^{0,1}(x)\right)$. Modified potential $w_{0, \infty}(x, z)$ presents the impact of asymptotic power $\Delta \varphi_{\infty}$ on the space potential distribution.

\section{Acknowledgement}

The important extension of the Bedeaux - Vlieger formal scheme into the larger one - for decomposing of different dimensional singularities, is due to Prof. B. Radoev (University of Sofia, Bulgaria, Dept. of Physical Chemistry). The author is grateful to Dr. Plamen Georgiev and Dr. Emil Molle (University of Sofia, Bulgaria, Faculty of Biology) for the useful comments on cell biology concepts and the assistance in preparing the illustrations.

This study was partially supported by grant No DDVU 02/90 of the Bulgarian National Science Foundation. 


\section{References}

[1] Ashcroft, N.W., Mermin, N.D., Solid States Physics, Holt, Rinehart and Winston - New York (1975).

[2] Bedeaux, D., Vlieger, J., Optical Properties of Surfaces, Imperial College Press, London(2001).

[3] Colton, D., Kress, R., Integral Equation Methods in Scattering Theory, John Wiley \& Sons, New York (1983).

[4] Cook, B., Kazakova, T., Madrid, P., Neal, J., Pauletti, M., Zhao, R., Cell-foreign Particle Interaction, IMA Preprint Series \# 2133-3 (Sept. 2006), Univ. of Minnesota.

[5] Ebert, Ph., Hun Chen, Heinrich, M., Simon, M., Urban, K., Lagally, M.G., Direct Determination of the Interaction between Vacancies on $\operatorname{InP}(110)$ Surfaces, Phys.Rev.Lett., 76, (№ 12), 18 March 1996.

[6] Gibbs, J., The Scientific Papers, 1, Dover, New York (1961).

[7] Heinrich, M., Ebert, Ph., Simon, M., Urban, K., Lagally, M.G., Temperature Dependent Vacancy Concentrations on $\operatorname{InP}(110)$ Surfaces, J. Vac. Sci. Technol. A. 13(3), May/Jun. 1995.

[8] Hörmander, L., The Analysis of Linear Partial Differential Operators, v. I-IV, SpringerVerlag, Berlin (1983).

[9] Israelishvili, J., Intermolecular and Surface Forces, Academic Press, London (1991).

[10] Jackson, J.D., Classical Electrodynamics, John Wiley \& Sons, New York, (1962).

[11] Junqueira, Z., Carneiro, J., Kelly, R.O., Basic Histology, A. ZANCE Medical Book (1995).

[12] Landau, L., Lifschitz, S., Lectures on Modern Physics, vol. VIII Electrodynamics of Solids, Nauka (Moskow) 1982 (in Russian).

[13] Radoev, B., Boev, T., Avramov, M. Electrostatics of Heterogeneous Monolayers, Adv. In Colloid and Interface Sci., 114-115 (2005) 93-101. 


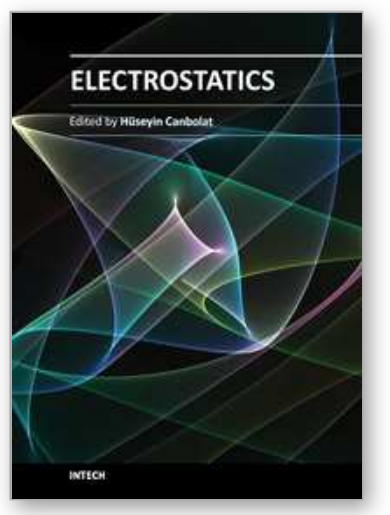

\author{
Electrostatics \\ Edited by Dr. Hüseyin Canbolat
}

ISBN 978-953-51-0239-7

Hard cover, 150 pages

Publisher InTech

Published online 14, March, 2012

Published in print edition March, 2012

In this book, the authors provide state-of-the-art research studies on electrostatic principles or include the electrostatic phenomena as an important factor. The chapters cover diverse subjects, such as biotechnology, bioengineering, actuation of MEMS, measurement and nanoelectronics. Hopefully, the interested readers will benefit from the book in their studies. It is probable that the presented studies will lead the researchers to develop new ideas to conduct their research.

\title{
How to reference
}

In order to correctly reference this scholarly work, feel free to copy and paste the following:

Toshko Boev (2012). Mathematical Models for Electrostatics of Heterogeneous Media, Electrostatics, Dr. Hüseyin Canbolat (Ed.), ISBN: 978-953-51-0239-7, InTech, Available from:

http://www.intechopen.com/books/electrostatics/mathematical-models-for-electrostatics-of-heterogeneousmedia

\section{INTECH}

open science | open minds

\section{InTech Europe}

University Campus STeP Ri

Slavka Krautzeka 83/A

51000 Rijeka, Croatia

Phone: +385 (51) 770447

Fax: +385 (51) 686166

www.intechopen.com

\section{InTech China}

Unit 405, Office Block, Hotel Equatorial Shanghai

No.65, Yan An Road (West), Shanghai, 200040, China 中国上海市延安西路65号上海国际贵都大饭店办公楼 405 单元 Phone: $+86-21-62489820$

Fax: +86-21-62489821 
(C) 2012 The Author(s). Licensee IntechOpen. This is an open access article distributed under the terms of the Creative Commons Attribution 3.0 License, which permits unrestricted use, distribution, and reproduction in any medium, provided the original work is properly cited. 